\title{
1 Construction of a solid Cox model for AML patients based 2 on multiomics bioinformatic analysis
}

$3 \mathrm{Fu} \mathrm{Li}^{1, \dagger}$, Jiao $\mathrm{Cai}^{2,3 \dagger}$, Jia Liu ${ }^{1}$, Shi-cang $\mathrm{Yu}^{3}$, Xi Zhang ${ }^{1}$, Yi Su ${ }^{2, *}$, Lei Gao ${ }^{1, *}$

$4{ }^{1}$ Medical Center of Hematology, Xinqiao Hospital, Army Medical University,

5 Chongqing, China

$6 \quad{ }^{2}$ Department of Hematology and Hematopoietic Stem Cell Transplantation Centre,

7 The General Hospital of Western Theater Command, Chengdu, China

$8{ }^{3}$ Department of Stem Cell and Regenerative Medicine, Southwest Hospital, Army

9 Medical University, Chongqing, China

$11{ }^{\#}$ Correspondence to:

12 Lei Gao: gaolei7765@163.com

14 Yi Su: suhang1234@hotmail.com

16 Competing interests: The authors declare that no competing interests exist. 


\section{Abstract}

Acute myeloid leukemia (AML) is a highly heterogeneous hematological malignancy. The bone marrow (BM) microenvironment in AML plays an important role in leukemogenesis, drug resistance and leukemia relapse. In this study, we aimed to identify reliable immune-related biomarkers for AML prognosis by multiomics analysis. We obtained expression profiles from The Cancer Genome Atlas (TCGA) database and constructed a LASSO-Cox regression model to predict the prognosis of AML using multiomics bioinformatic analysis data. This was followed by independent validation of the model in the GSE106291 ( $n=251)$, GSE12417 ( $n=163)$ and GSE37642 $(\mathrm{n}=137)$ datasets and mutated genes in clinical samples for predicting overall survival (OS). Molecular docking was performed to predict the most optimal ligands to these hub genes. The single-cell RNA sequence dataset GSE116256 was used to clarify the expression of the hub genes in different immune cell types. According to their significant differences in immune gene signatures and survival trends, we concluded that the immune infiltration-lacking subtype (IL type) is associated with better prognosis than the immune infiltration-rich subtype (IR type).

Using the LASSO model, we built a classifier based on 5 hub genes to predict the prognosis of AML $($ risk score $=-0.086 \times \mathrm{ADAMTS} 3+0.180 \times \mathrm{CD} 52+0.472 \times \mathrm{CLCN} 5$ $-0.356 \times \mathrm{HAL}+0.368 \times \mathrm{ICAM} 3)$. In summary, we constructed a prognostic model of AML using integrated multiomics bioinformatic analysis that could serve as a therapeutic classifier. 


\section{Introduction}

Acute myeloid leukemia (AML) is a highly heterogeneous group of hematological malignancies that are characterized by various cytogenetic and molecular heterogeneities (Dohner, Weisdorf, \& Bloomfield, 2015; Short, Rytting, \& Cortes, 2018). Although substantial progress has been achieved with combinatorial therapies including radiation, chemotherapy, immunotherapy and/or targeted therapy, the cure rate of patients remains only $35 \%-40 \%$ in younger patients (age $<60$ years) and 5\%-15\% in older patients (age $<60$ years)(Döhner et al., 2010). Relapse and refractory disease continue to be major obstacles in the treatment of AML, with 29\% or fewer patients living beyond 5 years.

Several studies have shown that changes in the bone marrow (BM) microenvironment in AML largely promote distinct biological processes in leukemogenesis, drug resistance and leukemia relapse(Ghobrial, Detappe, Anderson, \& Steensma, 2018). Thus, insights into BMM action may provide better diagnosis and treatment strategies for AML patients.

The BM microenvironment in AML is comprised of leukemia cells, stromal cells, endothelial cells and distinct immune cell subsets. Among them, vascular endothelial cells (ECs) promote leukemia cell proliferation, drug resistance, and recurrence through paracrine vascular endothelial growth factor (VEGF), adhesion, and fusion with leukemia cells, resulting in poor prognosis (Aguayo et al., 1999; Cogle et al., 2016; Padró et al., 2002; Wegiel, Ekberg, Talasila, Jalili, \& Persson, 2009). Stromal 
cells can promote chemotherapy resistance in leukemia cells through ligand-receptor interactions (Hazlehurst \& Dalton, 2001) such as SDF-1/CXCR4 (Tabe et al., 2007) and VLA-4/VCAM-1 (Jacamo et al., 2014). Adipocytes promote the proliferation, growth and chemotherapeutic resistance of leukemia cells by breaking down stored triglycerides into free fatty acids(Shafat et al., 2017) and secreting tumor-related proinflammatory cytokines (Ye et al., 2016). and suppression, leading to an imbalance of suppressor T cells and effector T cells, $\mathrm{T}$ cell exhaustion and an increase in myeloid-derived suppressor cells (MDSCs) and leukemia-supporting macrophages compared to normal bone marrow tissue(Mendez, Posey, \& Pandolfi, 2019). Recent studies on the characterization of the leukemia immune microenvironment could aid in the search for novel prognostic biomarkers and potential therapeutic targets (Vadakekolathu et al., 2020; Yan et al., 2019). In addition, treatments such as chemotherapy, immunotherapy, and combination therapy to alter the immune microenvironment of AML have been widely used (Allie, Zhang, Tsai, Noelle, \& Usherwood, 2013; Assi, Kantarjian, Ravandi, \& Daver, 2018). However, different immune cells have different effects in AML. Therefore, understanding the distribution and function of immune-related genes in different immune cells is of great significance to further explore the BM immune microenvironment of AML patients. 
genes on the prognosis of AML. We are the first group to screen for hub genes in this disease using multiomics analysis. We constructed a LASSO-Cox regression model to predict the prognosis of AML according to the characterization of the leukemia immune microenvironment. The distribution of hub genes in immune cells was revealed through single-cell sequencing data and may provide the potential for precise patient stratification and treatment.

\section{Materials and Methods}

\section{Datasets}

The test cohort of acute myelocytic leukemia (AML) was downloaded from The Cancer Genome Atlas (TCGA) database (https://www.cancer.gov/) and includes mRNA data from 151 cases (RNASeq V2), miRNA data from188 cases (Illumina HiSeq miRNAseq) and Illumina Human Methylation450 Bead Array data from 140 cases. Samples were selected for the study according to the following criteria: 1) acute myelocytic leukemia was pathologically diagnosed, 2) all three kinds of data were available for the patient, and 3) the clinical information was complete. Finally, 97 patients were selected for our following study.

The other datasets were obtained from the Gene Expression Omnibus (GEO) database (https://www.ncbi.nlm.nih.gov/geo/). A total of 4 AML patient datasets were employed as independent validation cohorts: GSE12417, containing 2 datasets in which 163 samples were analyzed using the GPL96 (Affymetrix Human Genome 
100

101

102

103

104

U133A Array) platform and 80 samples were analyzed using GPL570 (Affymetrix Human Genome U133 Plus 2.0 Array) platform; the GSE106291 dataset (251 samples), which was generated using the GPL18460 (Illumina HiSeq 1500, Homo sapiens) platform; and the GSE37642 dataset (137 samples), which was generated using the GPL570 (Affymetrix Human Genome U133 Plus 2.0 Array) platform. The single-cell RNA sequence dataset GSE116256, including 16 untreated samples (D0), was used to reveal the distribution of hub genes in immune cell types. The immune gene set, including 776 genes, was acquired from a previous study (Charoentong et al., 2017).

\section{Screening of candidate genes and hierarchical clustering}

Differential mRNA and miRNA expression were analyzed by the DESeq2 (Love, Huber, \& Anders, 2014) function $(P<0.05,|\log F C|>1)$. For each probe in the methylation data, the value shown is the $\beta$ value $(\beta=\mathrm{U} /(\mathrm{M}+\mathrm{U}+1))$, where $\mathrm{M}$ is the methylated probe signal strength and $U$ is the unmethylated probe signal value. The methylmix package (Gevaert, 2015) was used to analyze the correlation between the gene methylation level and mRNA expression value (Pearson correlation coefficient test, $\mathrm{R}>0.5, P<0.05$ ). Unsupervised hierarchical clustering was performed (Euclidean distances and complete linkage method) based on SIGs to establish an immunogenomic classification of TCGA-AML patients. 
The enrichment scores of 28 immune signatures in each AML sample were quantified using single-sample gene set enrichment analysis (ssGSEA) (Subramanian

et al., 2005). Stromal, immune, and estimate scores were further calculated to evaluate

MAlignant Tumor tissues using Expression data) algorithm (Yoshihara et al., 2013).

\section{Protein-protein interaction (PPI) network construction and gene ontology}

(GO) functional enrichment analysis

\section{Survival analysis and prognostic model construction}


Tibshirani, 2010; Simon, Friedman, Hastie, \& Tibshirani, 2011) was applied to

distinguish the most important SIGs and to construct a prognostic model for AML

based on a linear combination of the regression coefficients. Kaplan-Meier survival

validate the performance of the classifier.

\section{scRNA dataset analysis} hub genes in each immune cell type.

\section{Patients and samples}

A total of 55 patients with newly diagnosed AML were enrolled between Jan 2016 to Dec 2020 at the Xinqiao Hospital of the Army Medical University in China.

153 Samples were selected for the study according to the following criteria: 1) acute

154 myelocytic leukemia diagnoses were based on morphological findings, karyotype, and

155 immunophenotypic features of leukemia cells by consultant hematologist, 2) patients

156 were newly diagnosed, and remained untreated at the time of collection, 3) the clinical

157 information was complete, and 4) a total of 38 genes mutation and SNP were detected 158 using next-generation sequencing (NGS) technology. 
three-dimensional structure of CD52 (PDB ID: 6OBD), CLCN5 (PDB ID: 2J9L) and

ICAM3 (PDB ID: 1T0P) were retrieved from the Protein Data Bank

164

173 information for 97 samples from TCGA database (Table S1). A Cox proportional

(https://www.rcsb.org/). A library of $2115 \mathrm{FDA}$ approved compounds were extracted from ZINC15 druglike database (http://zinc.docking.org). The visualization of active interactions between proteins and compounds was performed by Discovery Studio Visualizer v4.5.0 (BIOVIA).

\section{affect patient prognosis}

\section{Classification of AML based on immune-related genes that significantly}

For a more extensive study of immune genes in AML, we retrieved transcriptome, microRNA, and DNA methylation profile data and integrated clinical hazard regression model was employed to analyze 776 immune-related genes (Charoentong et al., 2017) in the mRNA expression data of 97 samples $(P<0.05)$, and 98 survival-related immune genes (SIGs) that significantly affected the survival of AML patients were identified (Table S2).

Using unsupervised clustering analysis (Euclidean statistics and complete linkage method) of 98 SIGs, those 97 samples were clustered into three distinct 
bioRxiv preprint doi: https://doi org/10.1101/2021.09.15.460430 this version posted September 16 2021. The copyriaht holder has placed this preprint (which was not certified by peer review) in the Public Domain. It is no longer restricted by copyright. Anyone can legally share, reuse, remix, or adapt this material for any purpose without crediting the original authors.

180 immune subtypes (Im1: immune cluster 1, Im2: immune cluster 2, Im3: immune

181 cluster 3) based on the gene expression signature (Figure 1A). As shown in immune

183 but expressed at low levels in the Im2 cluster (Figure 1B). Kaplan-Meier survival

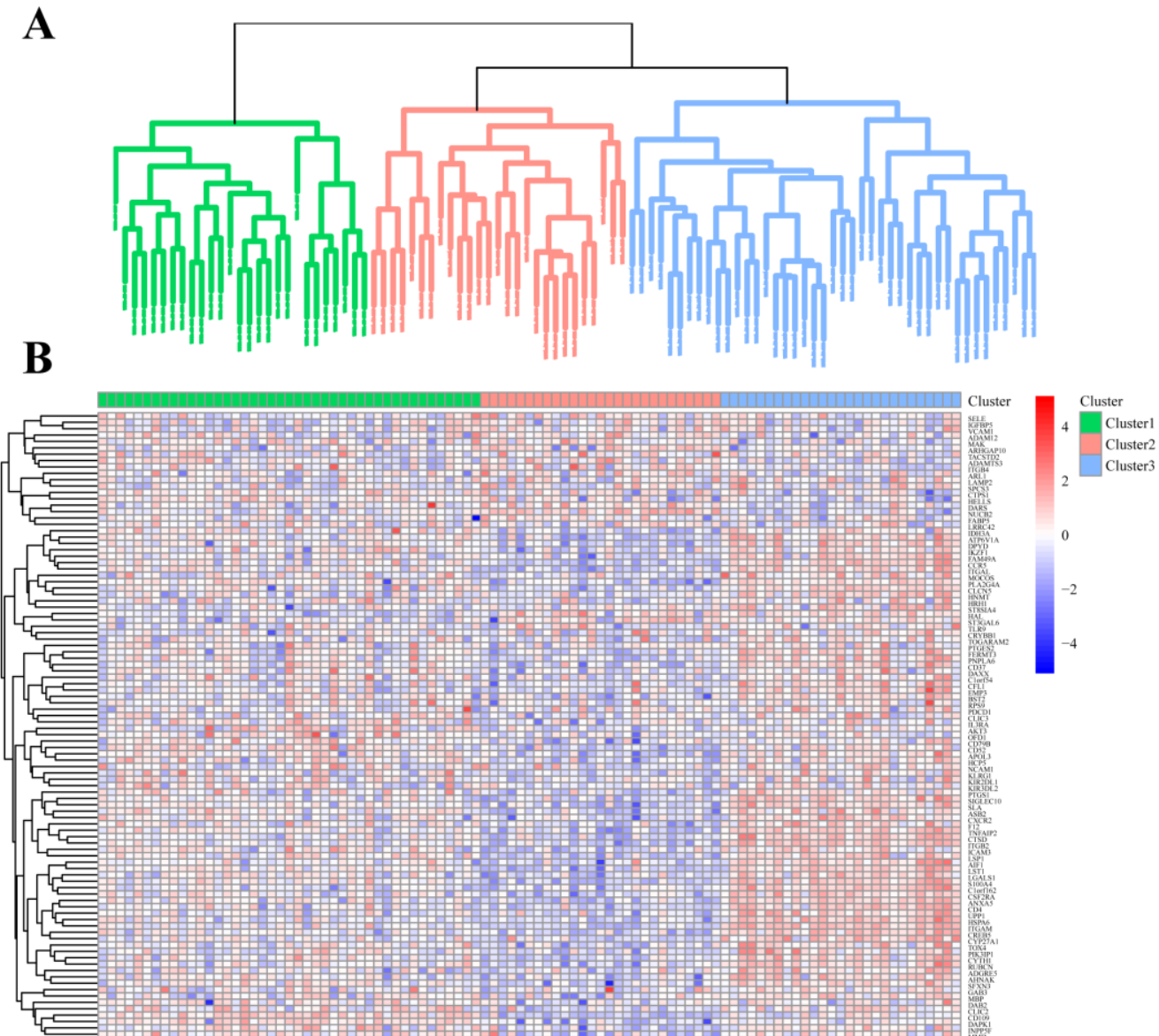

C

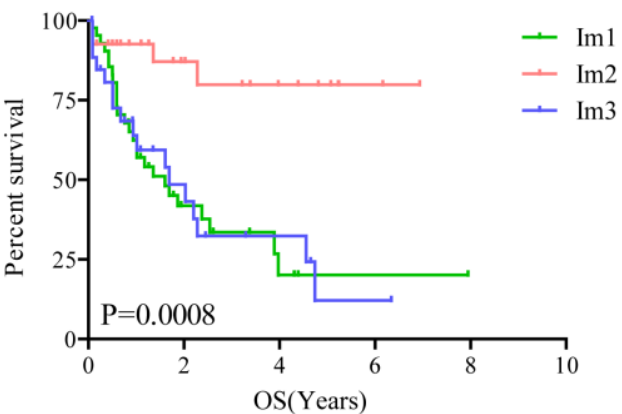

$10 / 44$ 
187 Figure 1. Unsupervised clustering analysis of AML patients based on 98 survival-related

188 immune genes. A. All 97 TCGA-AML patients were divided into 3 clusters (green: Im1

189 cluster, red: Im2 cluster, blue: Im3 cluster). B. Heatmap of 98 survival-related immune genes

190 in different AML clusters. C. The Kaplan-Meier survival analyses along with the Log-rank

191 test were used to compare the overall survival (OS) of the $\operatorname{Im} 1, \operatorname{Im} 2$ and $\operatorname{Im} 3$ clusters.

As the immune microenvironment was significantly correlated with the

199 findings demonstrated that the immune scores were significantly lower in the Im2 


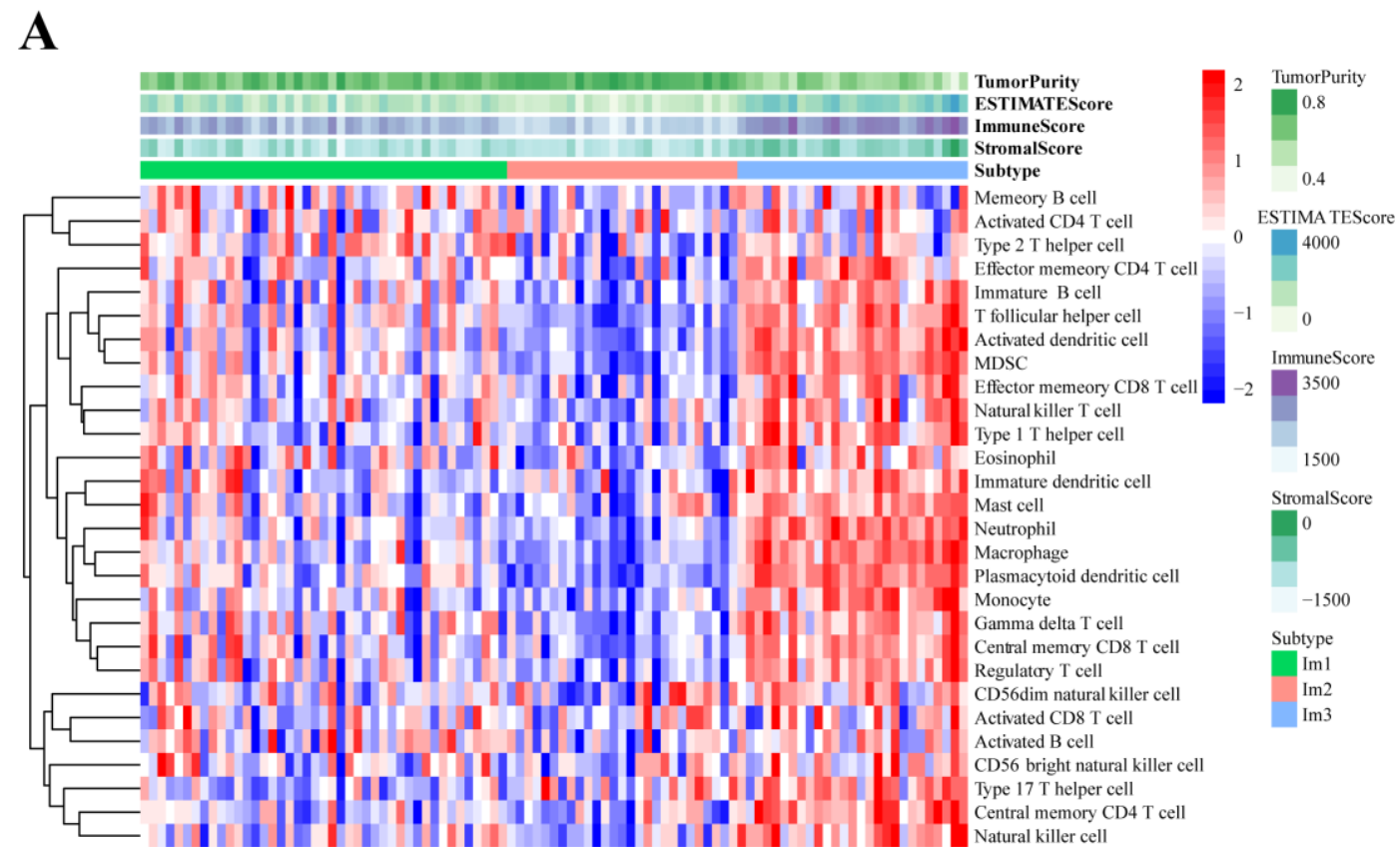

B

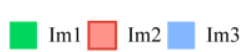

C

$\square \operatorname{Im} 1 \square \operatorname{Im} 2 \square \operatorname{Im} 3$
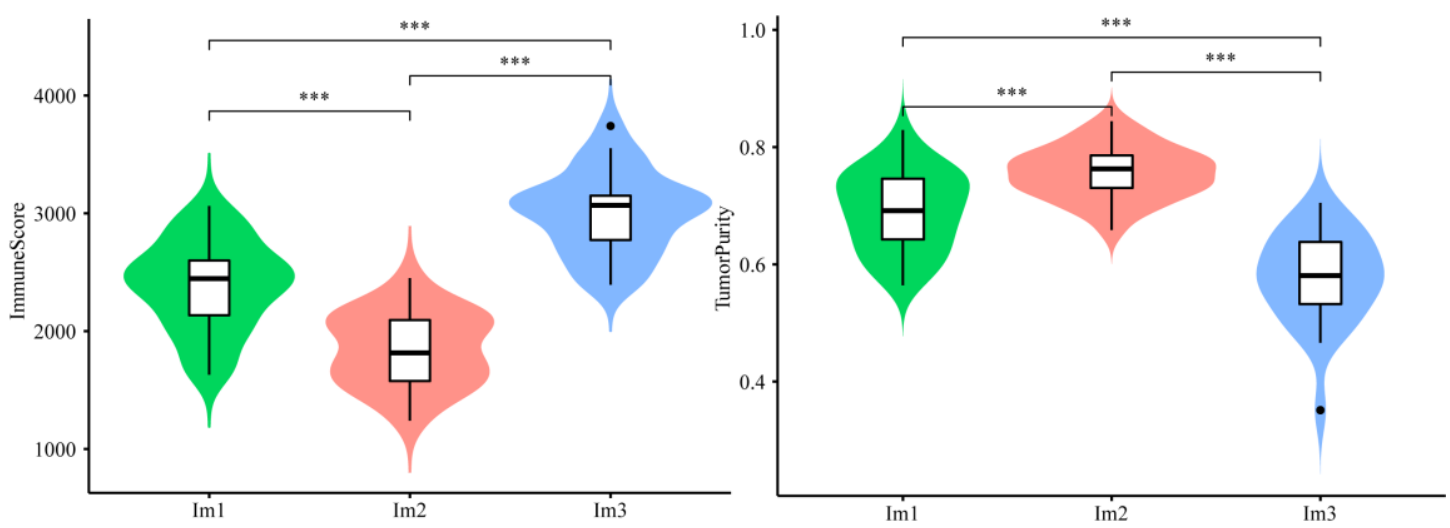

Figure 2. Immune functional characters of 3 AML patients clusters. A. Heatmap of the Im1, cohorts.

\section{DEG-SIGs were screened by mRNA expression data analyzing}


213 infiltration-lacking subtype (IL type) and $\operatorname{Im} 1 / 3$ as the immune infiltration-rich

214 subtype (IR type). To reveal the potential mechanisms of different prognoses between

IL and IR subtypes, an elaborate analysis of the mRNA expression profiles of the two

types of AML patients was implemented. We performed differentially expressed gene

analyses and identified 1936 differentially expressed genes (DEGs) with significant

differences between IL and IR subtypes. There were 42 SIG-DEGs which were common members of 1936 DEGs and 98 SIGs $(P<0.05$, $\mid$ Fold Change $\mid \geq 2)$ (Table 1)

(Figure 3A, B).

To elucidate the mechanism of prognosis difference between IL and IR subtype we analyzed the interaction on the STRING website (https://string-db.org/) and constructed protein-protein interaction (PPI) networks to draw visualized interactome maps of the 42 DEG-SIGs (Figure 3C). Gene ontology (GO) functional enrichment analysis distinguished some enriched terms in three subontologies: biological processes (BP), cellular component (CC), and molecular function (MF) (Figure 3D).

For BP, 42 DEG-SIGs were enriched in defense response, inflammatory response, and

228 immune system process. With regard to CC, 42 DEG-SIGs were enriched in integrin complex, external side of plasma membrane, and cell surface. For MF, 42 DEG-SIGs of AML patients. 


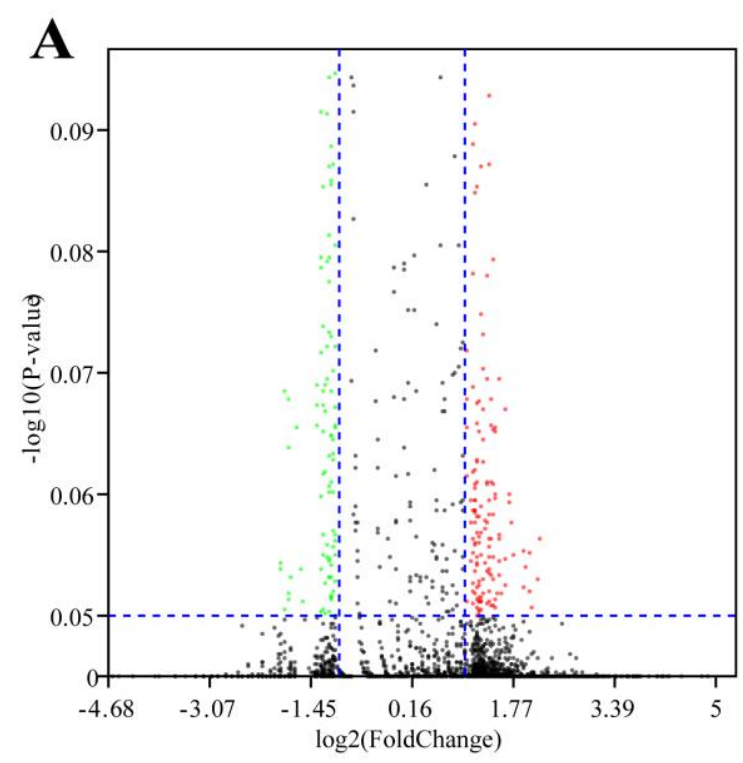

\section{B}

C

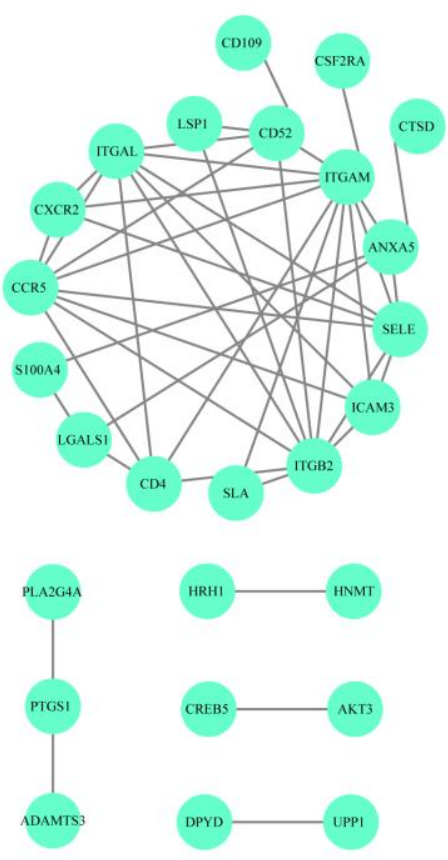

D

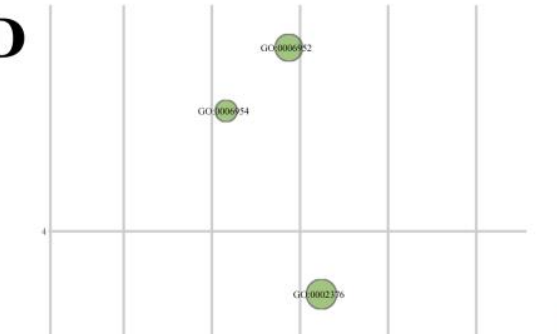

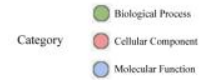

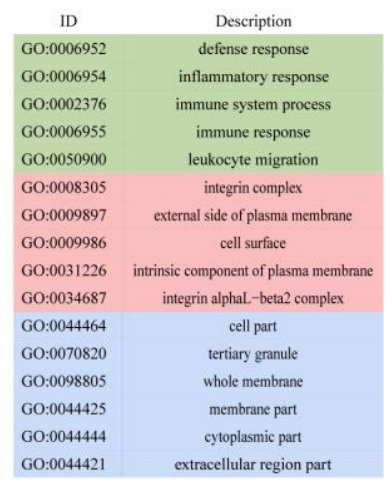

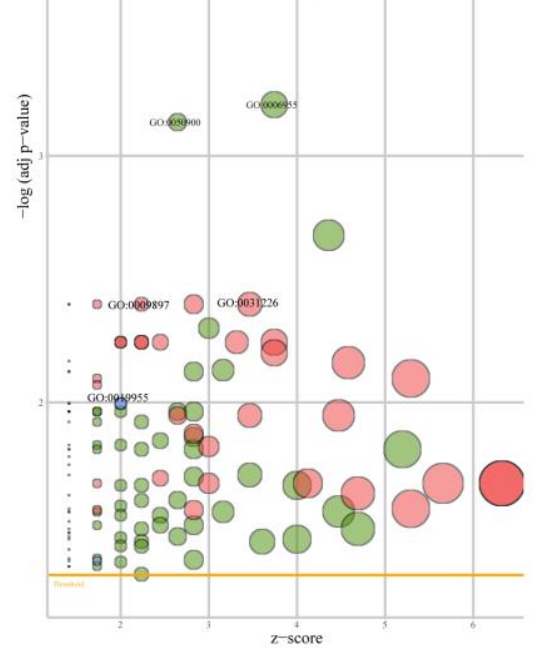

234 Figure 3. Difference analysis of the mRNA expression dataset from TCGA-AML patients. A.

235 Volcano plot of differentially expressed genes between immune infiltration-lacking subtype

236 (IL type) and immune infiltration-rich subtype (IR type). B. Venn plot of the intersecting

237 genes between differentially expressed genes (DEGs) and survival-related immune genes

238 (SIGs). C. PPI network of 42 overlap genes DEG-SIGs. D. The bubble plot represents the GO

$14 / 44$ 
239 functional enrichment analysis of 42 DEG-SIGs.

240 Table 1. Common 42 intersecting genes of differentially expressed genes (DEGs) and

241 survival-related immune genes (SIGs) between immune infiltration-lacking subtype (IL type)

242 and immune infiltration-rich subtype (IR type) $(\mathrm{P}<0.05$, $\mid$ Fold Change $\mid \geq 2)$.

\begin{tabular}{|c|c|c|}
\hline DEG-SIGs & Parametric p-value & Fold-change \\
\hline ADAMTS3 & 0.022973 & 0.37 \\
\hline AKT3 & 0.0002581 & 2.31 \\
\hline ANXA5 & 0.00000001 & 5.6 \\
\hline APOL3 & 0.00000009 & 3.11 \\
\hline ASB2 & 0.00000017 & 3.41 \\
\hline CCR5 & 0.00000025 & 5.73 \\
\hline CD109 & 0.00000033 & 18.68 \\
\hline $\mathrm{CD} 4$ & 0.0000012 & 3.11 \\
\hline CD52 & 0.00000041 & 4.64 \\
\hline CLCN5 & 0.0000017 & 2.78 \\
\hline CLIC2 & 0.0003049 & 2.5 \\
\hline CREB5 & 0.0000003 & 4.64 \\
\hline CSF2RA & 0.00000049 & 2.82 \\
\hline CTSD & 0.0000022 & 2.38 \\
\hline CXCR2 & 0.0000106 & 3.13 \\
\hline CYP27A1 & 0.0001964 & 4.2 \\
\hline DAPK1 & 0.00000057 & 3.52 \\
\hline DPYD & 0.00000065 & 2.97 \\
\hline $\mathrm{F} 12$ & 0.0000034 & 2.91 \\
\hline FAM49A & 0.0000331 & 2.5 \\
\hline HAL & 0.0014438 & 0.44 \\
\hline $\mathrm{HCP} 5$ & 0.0000109 & 2.31 \\
\hline HNMT & 0.045659 & 2.23 \\
\hline HRH1 & 0.0082122 & 2.03 \\
\hline HSPA6 & 0.0000058 & 3.28 \\
\hline ICAM3 & 0.0000006 & 2.08 \\
\hline ITGAL & 0.00000073 & 3.37 \\
\hline ITGAM & 0.00000081 & 4.61 \\
\hline ITGB2 & 0.00000089 & 3.93 \\
\hline LGALS1 & 0.0000022 & 3.18 \\
\hline LSP1 & 0.00000097 & 4.77 \\
\hline LST1 & 0.00000105 & 5.03 \\
\hline NUCB2 & 0.00000113 & 0.43 \\
\hline
\end{tabular}


PLA2G4A

PTGS1

S100A4

SELE

SFXN3

SIGLEC10

SLA

TNFAIP2

UPP1
0.00000121

0.0000015

0.00000129

0.0140379

0.00000137

0.0000046

0.00000145

0.00000153

0.000643
3.87

2.24

3.65

0.48

2.66

2.53

3.17

5.44

2.29

19 hub genes were screened by integrated analysis of mRNA expression data, miRNA expression data and methylation data next conducted integrated multiomics analysis to identify hub genes that were associated with prognosis. Comparing the miRNA expression profiles of patients between IL and IR subtypes, we revealed 93 miRNAs that were significantly differentially expressed $(P<0.05,|\mathrm{FC}| \geq 2)$ (Figure 4A). A total of 7294 target miRNA genes (TDEmiRs) were identified using DIANO TOOLS/microT-CDS

251 (threshold=0.9). Through integrated bioinformatics analysis, we selected 15 252 commonly differentially expressed genes from 42 DEG-SIGs and 7294 TDEmiRs 253 between the IL and IR subtypes (Figure 4C). significantly negative correlations between mRNA expression level and degree of 256 methylation for 355 genes $(\mathrm{R}<-0.5, \mathrm{p}<0.05)$. When these 355 methylation 257 correlation genes (MethylCor) were cross-referenced with the 42 DEG-SIGs, we 

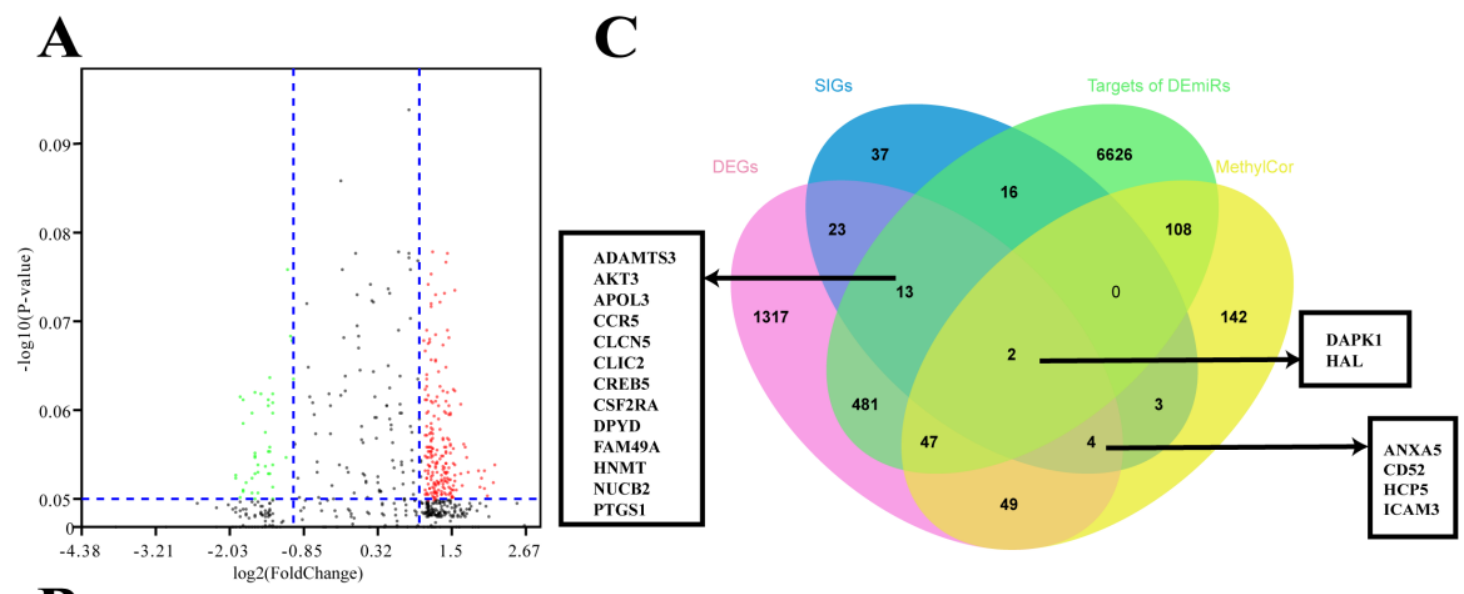

B

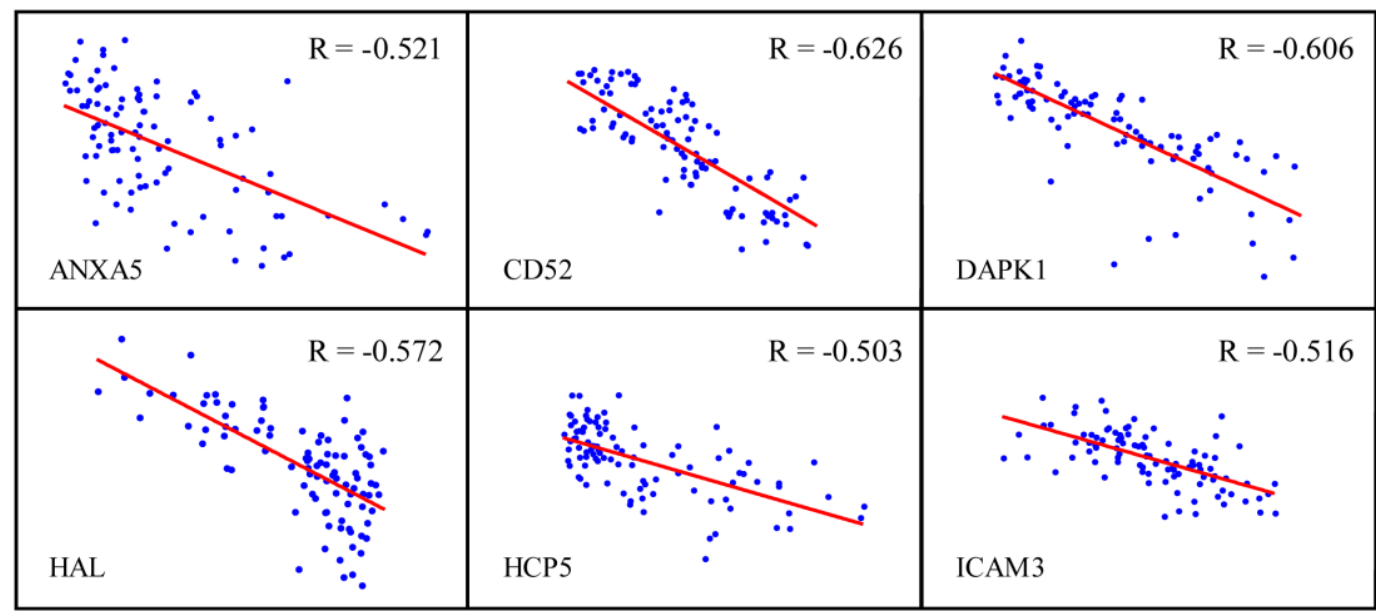

Figure 4. Multiomics analysis of 97 TCGA-AML patients. A. Volcano plot of differentially

DNA methylation level of 6 DEG \& MethylCor genes. C. Venn plot of the intersection of

DEGs, SIGs, targets of DEmiRs and MethylCor gene set.

\section{A prognostic model based on 5 hub genes was constructed}

Having observed significant differences in immune infiltration, gene expression 
270 built a classifier based on the 5 hub genes to predict the prognosis of AML (risk score

A

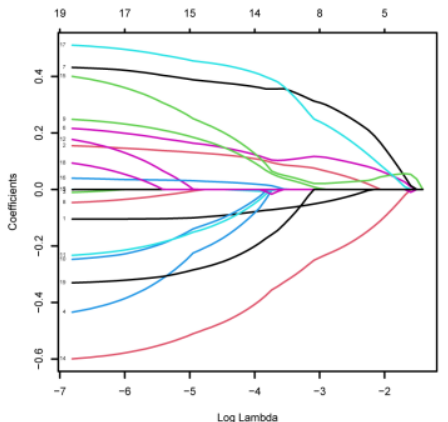

B

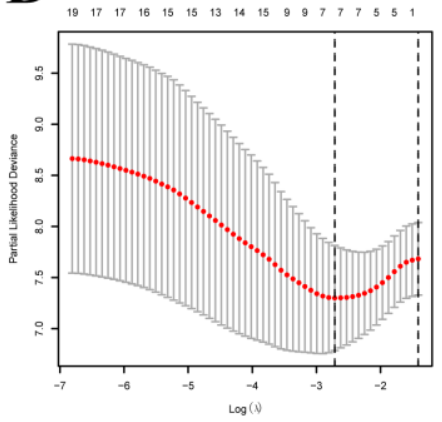

C

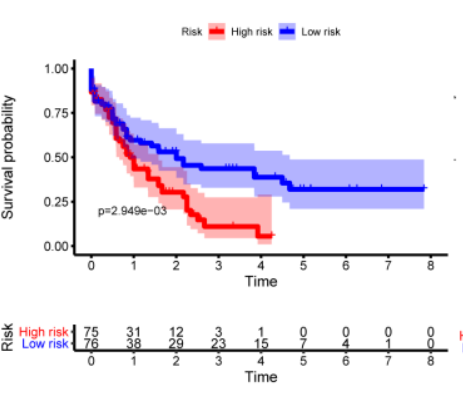

D

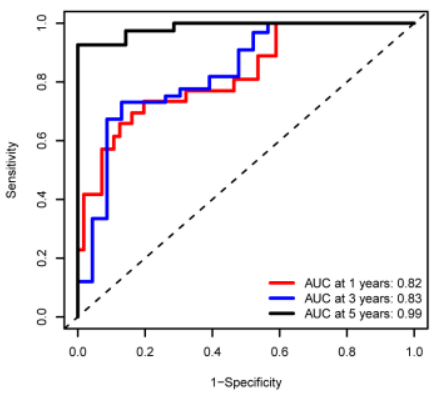

$\mathbf{E}$

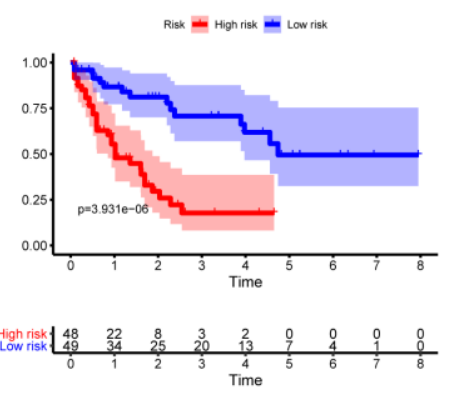

$\mathbf{F}$

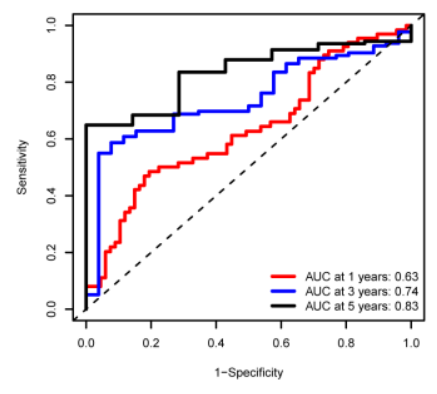

Figure 5. Construction of the COX regression model. A. LASSO coefficient profiles of 19 
281 lines were drawn at the optimal values determined by the minimum criteria and the 1-SE criteria. C, E. OS in patients with high vs. low risk scores depicted by Kaplan-Meier plots in the TCGA-AML-97 and TCGA-AML-151 cohorts. D, F. ROC curves depicting the accuracy of the Cox regression model in identifying AML subtypes with poor prognosis in the

TCGA-AML-97 and TCGA-AML-151 cohorts.

\section{The prognostic model can still predict the prognosis of AML patients}

effectively in various validation cohorts

Since APL can achieve good therapeutic effects with the application of ATRA and

ATO, we performed predictive tests separately in APL and non-APL patients.

Surprisingly, we observed the same conclusions in APL (log-rank test, $P=0.3618$,

(Figure 6A, B). To test this model further, validation cohorts were obtained from the

prognostic value of the 5-hub-gene-based model: GSE106291 (log-rank test,

$P=3.311 \times 10^{-06}, \quad$ AUC $\left.=0.66, \quad 0.66, \quad 0.61\right), \quad$ GSE12417-GPL96 $\quad$ log-rank test

$P=3.377 \times 10^{-02}, \quad$ AUC $=0.61, \quad 0.6, \quad$ NA),$\quad$ GSE12417-GPL570 (log-rank test,

$\left.P=3.763 \times 10^{-02}, \mathrm{AUC}=0.56,0.64, \mathrm{NA}\right)$, and GSE37642 $\left(\right.$ log-rank test, $P=3.966 \times 10^{-02}$, 
302 conventionally assigned risk groups and may guide clinical practice.

A

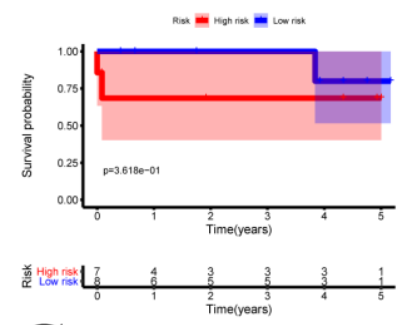

C

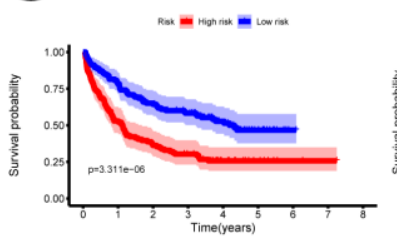

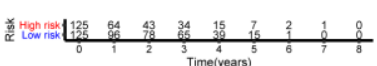

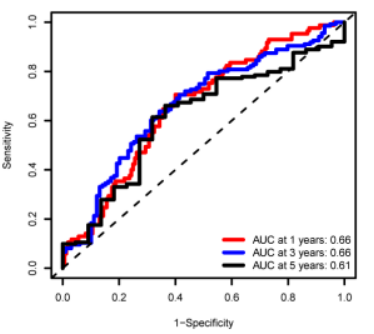

303

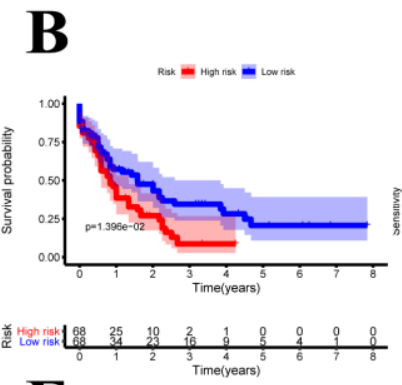

D
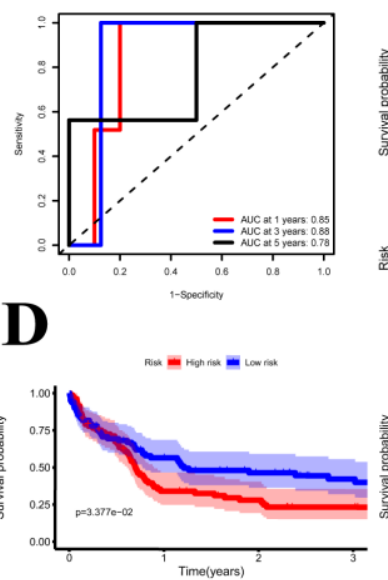

E
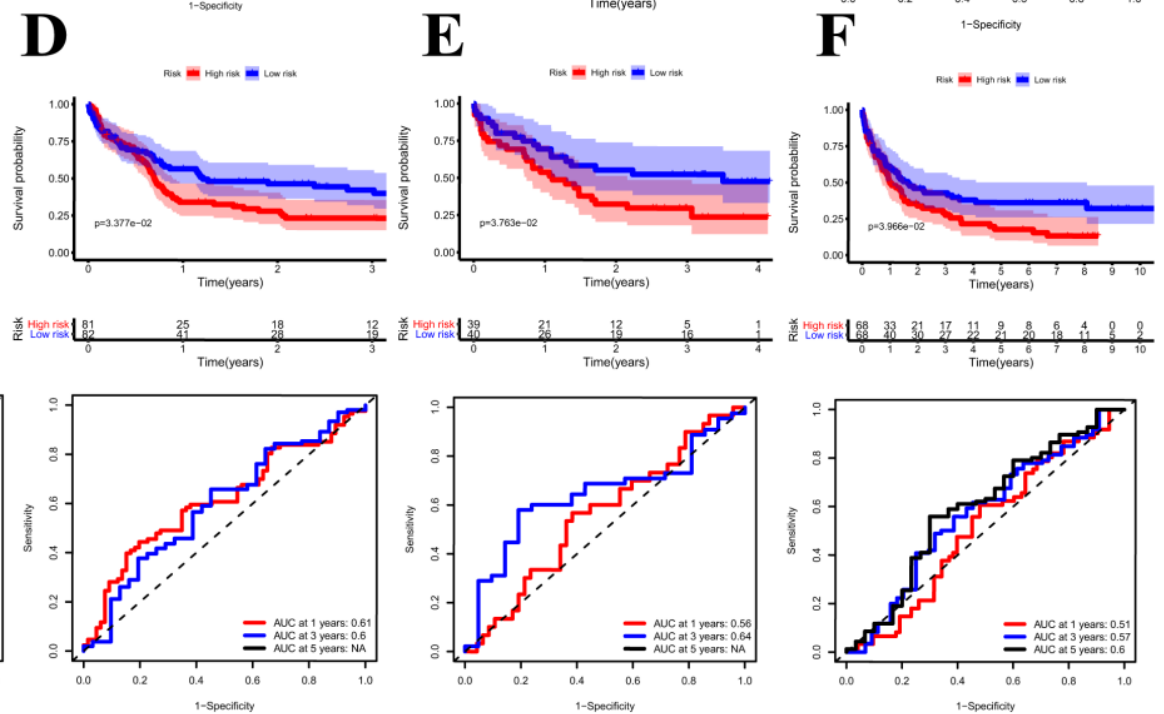

Figure 6. Validation of the prognostic model. A, B. Kaplan-Meier plots and ROC curves of

TCGA-AML-APL and TCGA-AML-nonAPL cohorts. C, D, E, F. Kaplan-Meier plots and

ROC curves of 3 GEO cohorts.

The better efficacy of the prognostic model for the prognosis of AML

patients was further verified by clinical samples

For verifying the prognostic value in the 5-hub-gene-based model, we collected 6575 genes mutation detected in 200 newly diagnosed AML patients (TCGA.LAML.mutect.somatic.maf, 
313 genes mutation detected in 55 newly diagnosed AML patients (Xinqiao Hostpital).

314 The common mutated genes were DNMT3A, IDH1, NRAS, RUNX1 and TET2. In this

315 model classification, the high risk was significantly associated with the mutation of

316 RUNX1 $(\mathrm{p}=0.015)$ and TET2 $(\mathrm{p}=0.054)$ considered by chi-square test (Table 2$)$.

317 Kaplan-Meier analyses of 55 patients with prognostic information indicated that

318 patients with mutation of RUNX1 (Figure 7A, $\mathrm{p}=0.0001$ ) and TET2 (Figure 7B,

$319 \mathrm{p}=0.2257)$ were correlated with a poor prognosis and had a shorter median survival

320 duration.

$\mathbf{A}$

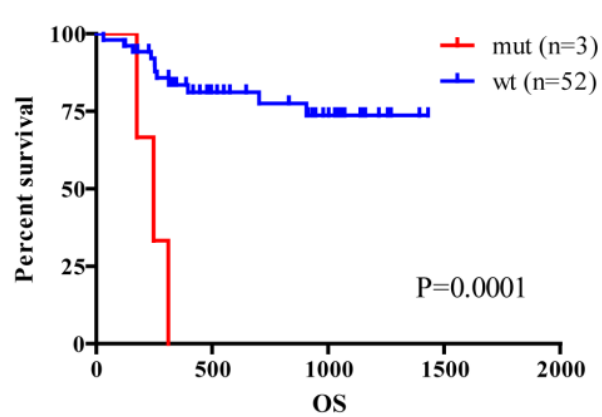

B

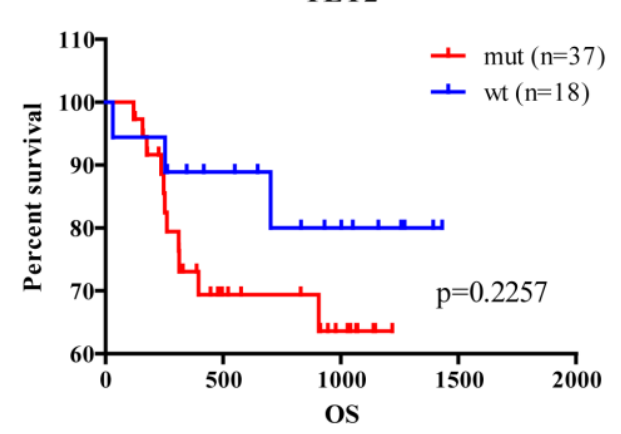

322

Figure 7. Analysis of hub genes and mutated genes in AML. A. Kaplan-Meier estimates of the OS according to RUNXI mutation status. B. Kaplan-Meier estimates of the OS according to TET2 mutation status. (wt: wild type, mut: mutation)

Table 2. Results of Chi-square test to 5 common mutated genes based on regrouping LASSO model in TCGA-AML database.

\begin{tabular}{c|cccc}
\hline & Regroup & Mutated sample size & Normal sample size & $\boldsymbol{P}$-value \\
\hline \multirow{2}{*}{ DNMT3A } & high & 12 & 40 & 0.326 \\
& low & 8 & 46 & \\
\hline$I D H 1$ & high & 4 & 48 & 0.201 \\
& low & 1 & 53 & \\
\hline
\end{tabular}




\begin{tabular}{c|cccc}
\hline \multirow{2}{*}{ NRAS } & high & 3 & 49 & 0.358 \\
& low & 1 & 53 & \\
\hline \multirow{2}{*}{ RUNX1 } & high & 8 & 44 & 0.015 \\
& low & 1 & 53 & 0.054 \\
\hline \multirow{2}{*}{ TET2 } & high & 4 & 48 & \\
& low & 0 & 54 & \\
\hline
\end{tabular}

The diverse distribution of hub genes in immune cells of AML patients

329 identified the single-cell sequencing dataset GSE116256 to describe the distribution

330 of the 5 hub genes in immune cells using the Seurat package for clustering and the

331 SingleR package for annotation (Figure 8A). As shown in the scatter plot (Figure 8B)

332 and violin plot (Figure 8C), CD52, ICAM3 and CLCN5 were widely expressed in

333 granulocytes, monocytes, T lymphocytes, B lymphocytes, dendritic cells and NK cells,

334 whereas ADAMTS3 was rarely expressed in those cells. HAL is highly expressed in

335 granulocytes and monocytes but rarely expressed in other immune cells. Accordingly,

336 we hypothesized that these hub genes play various roles through the regulation of

337 gene expression in specific cells. The hub gene expression of blood cells in the

338 Protein Atlas database (https://www.proteinatlas.org/) further confirmed this result 339 (Figure S3). 
bioRxiv preprint doi: https://doi.org/10.1101/2021.09.15.460430; this version posted September 16, 2021. The copyright holder has placed this preprint (which was not certified by peer review) in the Public Domain. It is no longer restricted by copyright. Anyone can legally share, reuse, remix, or adapt this material for any purpose without crediting the original authors.

A
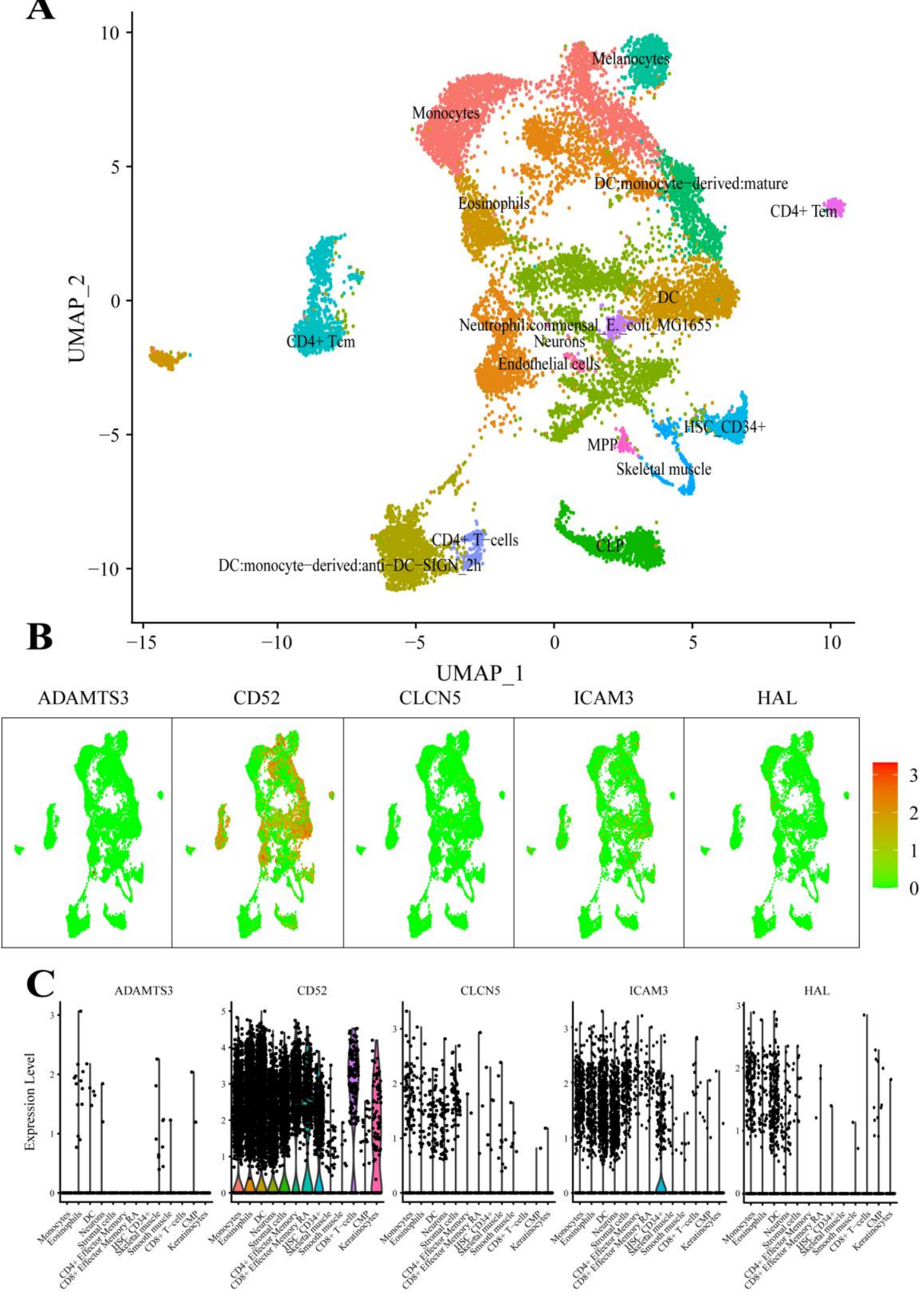
341 Figure 8. scRNA analysis of hub genes. A. Clustering analysis of the UMAP plot, color

342 coded based on cell types. B. Overlaying gene expression on UMAP clusters to illustrate the

343 distribution of hub genes in each cell type. C. Violin plots of hub genes in each cell type.

\section{Investigation of best-fitting compounds on hub genes}

To investigate best-fitting compounds, we performed virtual screening of molecular docking using the three-dimensional structure of CD52 (PDB ID: 6OBD),

347 CLCN5(PDB ID: 2J9L), ICAM3(PDB ID: 1T0P) and 2115 FDA approved 348 compounds in ZINC15 database. The predicted binding affinities of the top 2 hit 349 compounds against respective targets are ranked from highest to lowest. Binding 350 energy $(\mathrm{Kcal} / \mathrm{mol})$ for interaction of proteins and compounds are as follows: CD52 351 with ZINC164528615 (Glecaprevir) (-6.4 Kcal/mol), CD52 with ZINC3938684 352 (Toposar) $(-6.3 \mathrm{Kcal} / \mathrm{mol}) ; \quad$ ICAM3 with ZINC52955754 (Ergotamine) (-8.3 $353 \mathrm{Kcal} / \mathrm{mol})$, ICAM3 with ZINC1612996 (Irinotecan) $(-8.2 \mathrm{Kcal} / \mathrm{mol})$; CLCN5 with these proteins and candidate compounds are represented in Figure 9. 
A

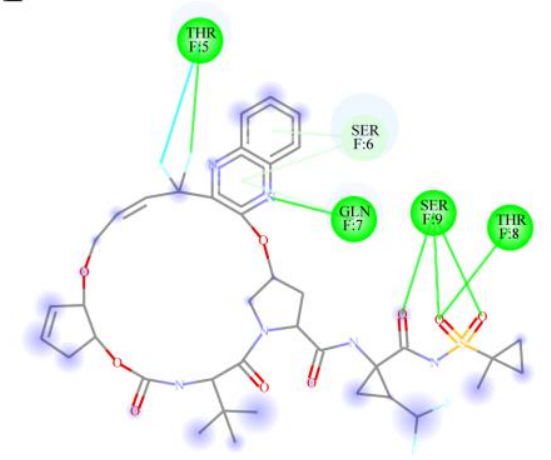

C

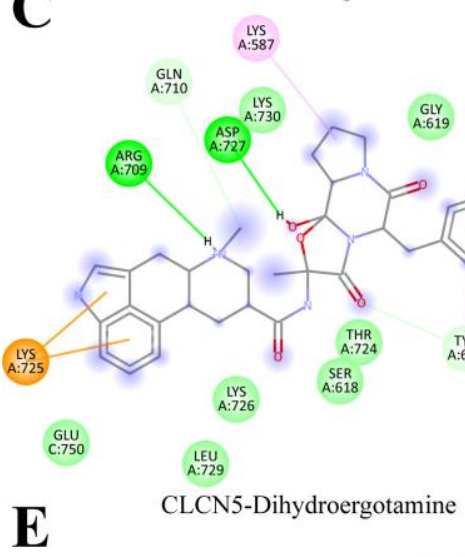

B

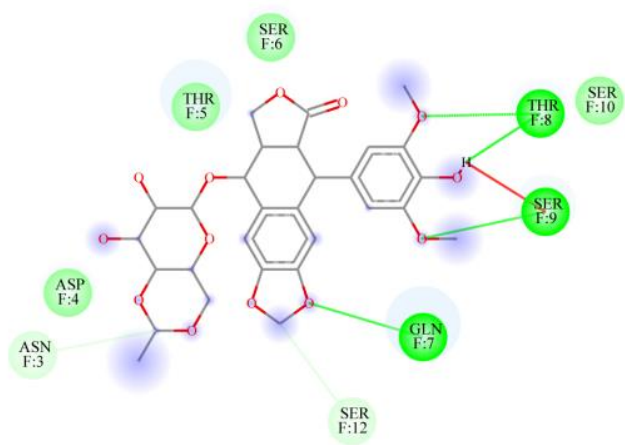

D

CD52-Toposar

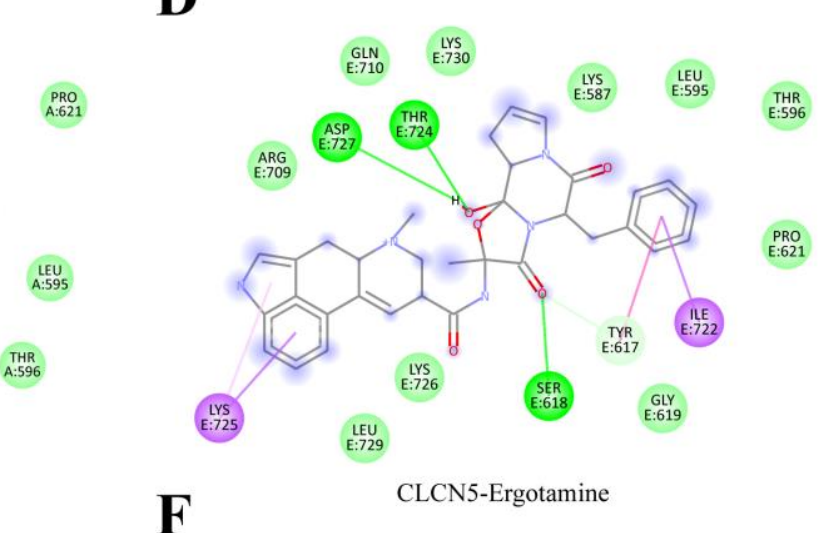

$\underset{\substack{\text { SER } \\ \mathrm{B}: 30}}{\mathrm{~B}}$
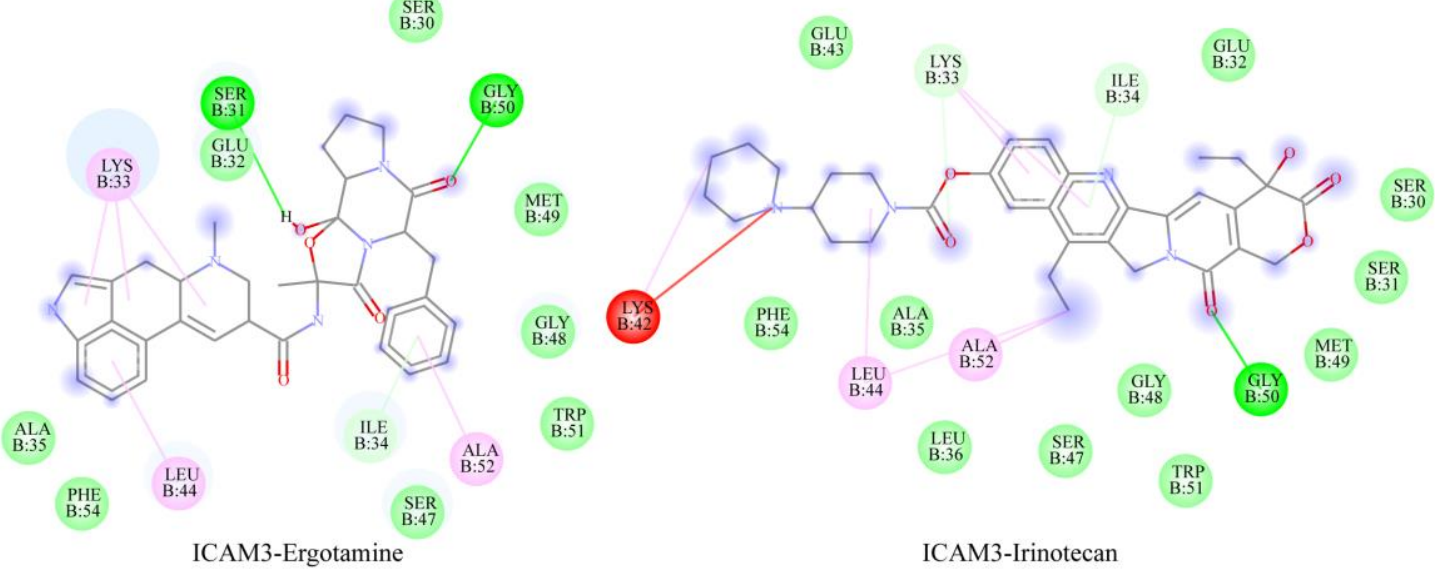

Figure 9. Shows 2D interaction representations of the best pose of A. CD52 with

ZINC164528615 (Glecaprevir), B. CD52 with ZINC3938684 (Toposar); C. ICAM3 with

ZINC52955754 (Ergotamine), D. ICAM3 with ZINC1612996 (Irinotecan); E. CLCN5 with

ZINC3978005 (Dihydroergotamine), F. CLCN5 with ZINC52955754 (Ergotamine).

\section{Discussion}

$25 / 44$ 
AML is a highly heterogeneous malignant tumor with poor prognosis. A large number of studies have demonstrated that the immune microenvironment of AML patients is altered significantly to promote leukemogenesis in AML(Mittal, Gubin, subtype) was significantly better than that of patients with immune infiltration enrichment (IR subtype), which was contrary to the effect of the immune infiltration

371 degree in solid tumors (Thorsson et al., 2018). Recent studies have shown that the heterogeneity of immune infiltration may be affected by the components of cytokines

373 in the microenvironment(Benci et al., 2016; J. Li et al., 2018) and the expression of 374 tumor driver genes(Bezzi et al., 2018). These heterogeneities were significantly 375 associated with immunotherapeutic effects.

377 found that the functions of genes with differential expression between the two 378 subtypes were mainly enriched in defense response, inflammatory response and 379 immune system process (BP); integrin complex, plasma membrane and cell surface 380 (CC); and cellular partial, tertiary granules, and whole membrane (MF). Consistent 381 with our results, several studies have confirmed that the biological functional diversity 382 of the immune system is significantly altered in the BM immune microenvironment. 
diverse omics data. $H u$ et al. constructed a DNA methylation-based prognostic model

for AML patients(Hu et al., 2019), but the accuracy of the model (AUC=0.67-0.75)

was lower than that of our model (AUC=0.82-0.99). A three-miRNA signature was

built for non-M3 AML patients by Xue et al., but due to the lack of validation by any

2017) was also less accurate ( $\mathrm{AUC}=0.66-0.71)$ than our model.

These conventional prognostic analyses generally consider only mRNA, miRNA,

three independent GEO-AML validation datasets confirm that the model is very

effective in predicting the prognosis of AML patients. The model consists of five hub

ADAMTS3 is a member of the ADAMTS family and plays an important role in the genesis and development of a variety of tumors(Gomis-Rüth, 2009; Rocks et al., 2008). Previous studies demonstrated that ADAMTS3 is expressed in mast cells(Charoentong et al., 2017), and mast cells have both tumor-promoting and 
ADAMTS3 expression was significantly negatively correlated with the prognosis of

406

407

408

AML and is rarely expressed in other immune cells. Therefore, we will pursue further in-depth analysis of how ADAMTS3 exerts its antitumor effect through mast cells to explore its potential therapeutic value.

CLCN5 (chloride voltage gated channel 5) is a member of the chloride channel family. It is widely expressed in a variety of tumor cells(Ernest, Weaver, Van Duyn, \& Sontheimer, 2005) and can enhance the chemotherapy resistance of chronic lymphocytic leukemia and multiple myeloma(Ruiz-Lafuente et al., 2015; Zhang et al., 2018). Hsa-let-7c-5p and hsa-mir-495-3p, two miRNAs that target CLCN5, were found to be involved in the occurrence and development of a variety of tumors(Liao et al., 2020; Wu et al., 2020). Our study confirmed that CLCN5 plays a role in promoting tumorigenesis in AML, but its specific mechanism needs deeper investigation.

HAL (histidine ammonia lyase) is the rate-limiting enzyme of histidine catabolism(Brand Lm Fau - Harper \& Harper, 1976). Kanarek et al. found that tumor cells with higher HAL expression levels possess higher sensitivity to methotrexate. Acute lymphocyte leukemia (ALL) patients with higher HAL expression were more likely to benefit from methotrexate treatment(Kanarek et al., 2018). In our study, we found that the expression level of HAL was significantly correlated with the degree of gene methylation. However, as a demethylation agent, hypomethylating agent (HMA) alone has difficulty achieving the intended effect in the clinical treatment of 
AML(Duchmann \& Itzykson, 2019). We speculated that the combination of methotrexate and HMA may achieve better efficacy when changes in HAL expression are detected. Surprisingly, we found that HAL was only expressed in granulocytes and monocytes. Consistent with AML cells, granulocytes and monocytes originate from myeloid precursor cells(Mendez et al., 2019). Therefore, insight into the methylation mechanism of HAL and its effect on the innate immune response and AML cells will be conducive to improving the therapeutic effect of demethylation agents. We also assessed the interaction between HAL and hsa-miR-582-3p, which has been previously indicated as a tumor suppressor miRNA in AML (H. Li et al., 2019). Thus, deeper investigation of the relationship between HAL and miR-582-3p will be helpful to further understand the potential mechanism of malignant progression of AML.

Our study found that CD52 was widely expressed in leukemia cells and all types of immune cells. Evidence has revealed the high expression of CD52 in CD34+ stem cells of AML (5q-) patients and a significantly negative correlation between the CD52 expression value and the prognosis of AML (Blatt et al., 2014), which is consistent with our results. ICAM-3 (intercellular adhesion molecule 3, CD50) belongs to the ICAM (intercellular adhesion molecule) immunoglobulin superfamily and plays important roles in immune response and tumor development(Cavallaro \& Christofori, 2004; Xiao, Mruk, \& Cheng, 2013). Consistent with our scRNA sequencing results, ICAM-3 is expressed in granulocytes, monocytes and lymphocytes(de Fougerolles \& Springer, 1992). In vivo and in vitro experiments confirmed that ICAM-3 participates 
447 in the proliferation, stemness and radiotherapy resistance of various tumors through

448 the FAK pathway, PI3K/Akt pathway or other mechanisms(Chung et al., 2005; Kim et

449 al., 2006; Shen et al., 2018). Interestingly, we found that the expression levels of

450 CD52 and ICAM-3 were both significantly correlated with the degree of methylation

451 but had opposite impacts on the prognosis of AML patients. Therefore, the roles of

452 CD52 and ICAM-3 should be considered in HMA reagent treatment.

In conclusion, using a multiomics analysis and validation approach, we

454 constructed and validated a novel, 5-hub-gene-based model that allows robust risk

455 stratification and facilitates the identification of prognosis in AML. The distribution of

456 the 5 hub genes in immune cells was revealed through scRNA sequencing analysis.

457 Furthermore, we preliminarily explored the possible mechanisms of these hub genes

458 in the leukemogenesis of AML. These findings may provide novel therapeutic targets

459 for AML. Our study also provided new insight into the use of demethylation drugs in

460 AML. Of course, this study is limited to bioinformatics analysis, and the proposed

461 approaches need to be further tested in the clinic. 


\section{References}

463

464

465

466

467

468

469

470

471

472

473

474

475

476

477

478

479

480

481

482

483

484

485

486

487

488

489

490

491

492

493

494

495

496

497

498

499

500

501

502

503

Aguayo, A., Estey, E., Kantarjian, H., Mansouri, T., Gidel, C., Keating, M., . . . Albitar, M. (1999). Cellular vascular endothelial growth factor is a predictor of outcome in patients with acute myeloid leukemia. Blood, 94(11), 3717-3721. doi: 10.1182/blood.V94.11.3717

Allie, S. R., Zhang, W., Tsai, C. Y., Noelle, R. J., \& Usherwood, E. J. (2013). Critical role for all-trans retinoic acid for optimal effector and effector memory CD8 T cell differentiation. J Immunol, 190(5), 2178-2187. doi: 10.4049/jimmunol.1201945

Aran, D., Looney, A. P., Liu, L., Wu, E., Fong, V., Hsu, A., . . . Bhattacharya, M. (2019). Reference-based analysis of lung single-cell sequencing reveals a transitional profibrotic macrophage. Nature Immunology, 20(2), 163-172. doi: 10.1038/s41590-018-0276-y

Assi, R., Kantarjian, H., Ravandi, F., \& Daver, N. (2018). Immune therapies in acute myeloid leukemia: a focus on monoclonal antibodies and immune checkpoint inhibitors. Curr Opin Hematol, 25(2), 136-145. doi: 10.1097/moh.0000000000000401

Benci, J. L., Xu, B., Qiu, Y., Wu, T. J., Dada, H., Twyman-Saint Victor, C., . . Minn, A. J. (2016). Tumor Interferon Signaling Regulates a Multigenic Resistance Program to Immune Checkpoint Blockade. Cell, 167(6), 1540-1554.e1512. doi: 10.1016/j.cell.2016.11.022

Bezzi, M., Seitzer, N., Ishikawa, T., Reschke, M., Chen, M., Wang, G., . . Pandolfi, P. P. (2018). Diverse genetic-driven immune landscapes dictate tumor progression through distinct mechanisms. Nat Med, 24(2), 165-175. doi: 10.1038/nm.4463

Blatt, K., Herrmann, H., Hoermann, G., Willmann, M., Cerny-Reiterer, S., Sadovnik, I., . . Valent, P. (2014). Identification of campath-1 (CD52) as novel drug target in neoplastic stem cells in 5q-patients with MDS and AML. Clin Cancer Res, 20(13), 3589-3602. doi: 10.1158/1078-0432.Ccr-13-2811

Brand Lm Fau - Harper, A. E., \& Harper, A. E. (1976). Studies on the production and assessment of experimental histidinemia in the rat. Biochim Biophys Acta, 444(1)(0006-3002 (Print)), 294-306. doi: 10.1016/0304-4165(76)90246-4

Butler, A., Hoffman, P., Smibert, P., Papalexi, E., \& Satija, R. (2018). Integrating single-cell transcriptomic data across different conditions, technologies, and species. Nature Biotechnology, 36(5), 411-420. doi: 10.1038/nbt.4096

Cavallaro, U., \& Christofori, G. (2004). Cell adhesion and signalling by cadherins and Ig-CAMs in cancer. Nat Rev Cancer, 4(2), 118-132. doi: 10.1038/nrc1276

Charoentong, P., Finotello, F., Angelova, M., Mayer, C., Efremova, M., Rieder, D., . . Trajanoski, Z. (2017). Pan-cancer Immunogenomic Analyses Reveal Genotype-Immunophenotype Relationships and Predictors of Response to Checkpoint Blockade. Cell Rep, 18(1), 248-262. doi: 10.1016/j.celrep.2016.12.019

Chung, Y. M., Kim, B. G., Park, C. S., Huh, S. J., Kim, J., Park, J. K., . . Bae, D. S. (2005). Increased expression of ICAM-3 is associated with radiation resistance in cervical cancer. Int $J$ Cancer, 117(2), 194-201. doi: 10.1002/ijc.21180

Cogle, C. R., Bosse, R. C., Brewer, T., Migdady, Y., Shirzad, R., Kampen, K. R., \& Saki, N. (2016). Acute myeloid leukemia in the vascular niche. Cancer Lett, 380(2), 552-560. doi: 10.1016/j.canlet.2015.05.007

Döhner, H., Estey Eh Fau - Amadori, S., Amadori S Fau - Appelbaum, F. R., Appelbaum Fr Fau $31 / 44$ 
Büchner, T., Büchner T Fau - Burnett, A. K., Burnett Ak Fau - Dombret, H., . . Bloomfield, C. D. (2010). Diagnosis and management of acute myeloid leukemia in adults: recommendations from an international expert panel, on behalf of the European LeukemiaNet. Blood, 115(3)(1528-0020 (Electronic)), 453-474. doi: 10.1182/blood-2009-07-235358

de Fougerolles, A. R., \& Springer, T. A. (1992). Intercellular adhesion molecule 3, a third adhesion counter-receptor for lymphocyte function-associated molecule 1 on resting lymphocytes. $J$ Exp Med, 175(1)(0022-1007 (Print)), 185-190. doi: 10.1084/jem.175.1.185

Dohner, H., Weisdorf, D. J., \& Bloomfield, C. D. (2015). Acute Myeloid Leukemia. N Engl J Med, 373(12), 1136-1152. doi: 10.1056/NEJMra1406184

Duchmann, M., \& Itzykson, R. (2019). Clinical update on hypomethylating agents. Int J Hematol, 110(2), 161-169. doi: 10.1007/s12185-019-02651-9

Ernest, N. J., Weaver, A. K., Van Duyn, L. B., \& Sontheimer, H. W. (2005). Relative contribution of chloride channels and transporters to regulatory volume decrease in human glioma cells. Am J Physiol Cell Physiol, 288(6), C1451-1460. doi: 10.1152/ajpcell.00503.2004

Fleischmann, A., Schlomm, T., Köllermann, J., Sekulic, N., Huland, H., Mirlacher, M., . . . Erbersdobler, A. (2009). Immunological microenvironment in prostate cancer: high mast cell densities are associated with favorable tumor characteristics and good prognosis. Prostate, 69(9), 976-981. doi: 10.1002/pros.20948

Friedman, J., Hastie, T., \& Tibshirani, R. (2010). Regularization Paths for Generalized Linear Models via Coordinate Descent. J Stat Softw, 33(1), 1-22. doi: 10.18637/jss.v033.i01

Gevaert, O. (2015). MethylMix: an R package for identifying DNA methylation-driven genes. Bioinformatics, 31(11), 1839-1841. doi: 10.1093/bioinformatics/btv020

Ghobrial, I. M., Detappe, A., Anderson, K. C., \& Steensma, D. P. (2018). The bone-marrow niche in MDS and MGUS: implications for AML and MM. Nat Rev Clin Oncol, 15(4), 219-233. doi: 10.1038/nrclinonc.2017.197

Gomis-Rüth, F. X. (2009). Catalytic domain architecture of metzincin metalloproteases. J Biol Chem, 284(23), 15353-15357. doi: 10.1074/jbc.R800069200

Groot Kormelink, T., Abudukelimu, A., \& Redegeld, F. A. (2009). Mast cells as target in cancer therapy. Curr Pharm Des, 15(16), 1868-1878. doi: 10.2174/138161209788453284

Hazlehurst, L. A., \& Dalton, W. S. (2001). Mechanisms associated with cell adhesion mediated drug resistance (CAM-DR) in hematopoietic malignancies. Cancer Metastasis Rev, 20(1-2), 43-50. doi: 10.1023/a:1013156407224

Hu, L., Gao, Y., Shi, Z., Liu, Y., Zhao, J., Xiao, Z., . . . Tong, X. (2019). DNA methylation-based prognostic biomarkers of acute myeloid leukemia patients. Ann Transl Med, 7(23), 737. doi: 10.21037/atm.2019.11.122

Huang da, W., Sherman, B. T., \& Lempicki, R. A. (2009a). Bioinformatics enrichment tools: paths toward the comprehensive functional analysis of large gene lists. Nucleic Acids Res, 37(1), 1-13. doi: $10.1093 /$ nar/gkn923

Huang da, W., Sherman, B. T., \& Lempicki, R. A. (2009b). Systematic and integrative analysis of large gene lists using DAVID bioinformatics resources. Nat Protoc, 4(1), 44-57. doi: 10.1038/nprot.2008.211

Huang, R., Liao, X., \& Li, Q. (2017). Identification and validation of potential prognostic gene biomarkers for predicting survival in patients with acute myeloid leukemia. OncoTargets and 
therapy, 10, 5243-5254. doi: 10.2147/OTT.S147717

Jacamo, R., Chen, Y., Wang, Z., Ma, W., Zhang, M., Spaeth, E. L., . . Andreeff, M. (2014). Reciprocal leukemia-stroma VCAM-1/VLA-4-dependent activation of NF- $\kappa \mathrm{B}$ mediates chemoresistance. Blood, 123(17), 2691-2702. doi: 10.1182/blood-2013-06-511527

Kanarek, N., Keys, H. R., Cantor, J. R., Lewis, C. A., Chan, S. H., Kunchok, T., . . Sabatini, D. M. (2018). Histidine catabolism is a major determinant of methotrexate sensitivity. Nature, 559(7715), 632-636. doi: 10.1038/s41586-018-0316-7

Kim, Y. G., Kim, M. J., Lim, J. S., Lee, M. S., Kim, J. S., \& Yoo, Y. D. (2006). ICAM-3-induced cancer cell proliferation through the PI3K/Akt pathway. Cancer Lett, 239(1), 103-110. doi: 10.1016/j.canlet.2005.07.023

Li, H., Tian, X., Wang, P., Huang, M., Xu, R., \& Nie, T. (2019). MicroRNA-582-3p negatively regulates cell proliferation and cell cycle progression in acute myeloid leukemia by targeting cyclin B2. Cell Mol Biol Lett, 24, 66. doi: 10.1186/s11658-019-0184-7

Li, J., Byrne, K. T., Yan, F., Yamazoe, T., Chen, Z., Baslan, T., . . . Stanger, B. Z. (2018). Tumor Cell-Intrinsic Factors Underlie Heterogeneity of Immune Cell Infiltration and Response to Immunotherapy. Immunity, 49(1), 178-193.e177. doi: 10.1016/j.immuni.2018.06.006

Liao, K., Qian, Z., Zhang, S., Chen, B., Li, Z., Huang, R., . . Lin, Y. (2020). The LGMN pseudogene promotes tumor progression by acting as a miR-495-3p sponge in glioblastoma. Cancer Lett, 490, 111-123. doi: 10.1016/j.canlet.2020.07.012

Love, M. I., Huber, W., \& Anders, S. (2014). Moderated estimation of fold change and dispersion for RNA-seq data with DESeq2. Genome Biol, 15(12), 550. doi: 10.1186/s13059-014-0550-8

Mendez, L. M., Posey, R. R., \& Pandolfi, P. P. (2019). The Interplay Between the Genetic and Immune Landscapes of AML: Mechanisms and Implications for Risk Stratification and Therapy. Front Oncol, 9, 1162. doi: 10.3389/fonc.2019.01162

Mittal, D., Gubin, M. M., Schreiber, R. D., \& Smyth, M. J. (2014). New insights into cancer immunoediting and its three component phases--elimination, equilibrium and escape. Curr Opin Immunol, 27, 16-25. doi: 10.1016/j.coi.2014.01.004

Oldford, S. A., \& Marshall, J. S. (2015). Mast cells as targets for immunotherapy of solid tumors. Mol Immunol, 63(1), 113-124. doi: 10.1016/j.molimm.2014.02.020

Padró, T., Bieker, R., Ruiz, S., Steins, M., Retzlaff, S., Bürger, H., . . . Mesters, R. M. (2002). Overexpression of vascular endothelial growth factor (VEGF) and its cellular receptor KDR (VEGFR-2) in the bone marrow of patients with acute myeloid leukemia. Leukemia, 16(7), 1302-1310. doi: 10.1038/sj.leu.2402534

Rajput, A. B., Turbin, D. A., Cheang, M. C., Voduc, D. K., Leung, S., Gelmon, K. A., . . Huntsman, D. G. (2008). Stromal mast cells in invasive breast cancer are a marker of favourable prognosis: a study of 4,444 cases. Breast Cancer Res Treat, 107(2), 249-257. doi: 10.1007/s10549-007-9546-3

Rocks, N., Paulissen, G., El Hour, M., Quesada, F., Crahay, C., Gueders, M., . . Cataldo, D. (2008). Emerging roles of ADAM and ADAMTS metalloproteinases in cancer. Biochimie, 90(2), 369-379. doi: 10.1016/j.biochi.2007.08.008

Ruiz-Lafuente, N., Alcaraz-Garcia, M. J., Sebastian-Ruiz, S., Garcia-Serna, A. M., Gomez-Espuch, J., Moraleda, J. M., . . Parrado, A. (2015). IL-4 Up-Regulates MiR-21 and the MiRNAs Hosted in the CLCN5 Gene in Chronic Lymphocytic Leukemia. PLoS One, 10(4), e0124936. doi: 


\subsection{1/journal.pone. 0124936}

Shafat, M. S., Oellerich, T., Mohr, S., Robinson, S. D., Edwards, D. R., Marlein, C. R., . . Rushworth, S. A. (2017). Leukemic blasts program bone marrow adipocytes to generate a protumoral microenvironment. Blood, 129(10), 1320-1332. doi: 10.1182/blood-2016-08-734798

Shannon, P., Markiel, A., Ozier, O., Baliga, N. S., Wang, J. T., Ramage, D., . . Ideker, T. (2003). Cytoscape: A Software Environment for Integrated Models of Biomolecular Interaction Networks. Genome Research, 13(11), 2498-2504. doi: 10.1101/gr.1239303

Shen, W., Xie, J., Zhao, S., Du, R., Luo, X., He, H., . . Luo, Y. (2018). ICAM3 mediates inflammatory signaling to promote cancer cell stemness. Cancer Lett, 422, 29-43. doi: 10.1016/j.canlet.2018.02.034

Short, N. J., Rytting, M. E., \& Cortes, J. E. (2018). Acute myeloid leukaemia. The Lancet, 392(10147), 593-606. doi: https://doi.org/10.1016/S0140-6736(18)31041-9

Simon, N., Friedman, J., Hastie, T., \& Tibshirani, R. (2011). Regularization Paths for Cox's Proportional Hazards Model via Coordinate Descent. J Stat Softw, 39(5), 1-13. doi: 10.18637/jss.v039.i05

Stuart, T., Butler, A., Hoffman, P., Hafemeister, C., Papalexi, E., Mauck, W. M., III, . . Satija, R. (2019). Comprehensive Integration of Single-Cell Data. Cell, 177(7), 1888-1902.e1821. doi: 10.1016/j.cell.2019.05.031

Subramanian, A., Tamayo, P., Mootha, V. K., Mukherjee, S., Ebert, B. L., Gillette, M. A., . . Mesirov, J. P. (2005). Gene set enrichment analysis: a knowledge-based approach for interpreting genome-wide expression profiles. Proc Natl Acad Sci U S A, 102(43), 15545-15550. doi: 10.1073/pnas.0506580102

Szklarczyk, D., Gable, A. L., Lyon, D., Junge, A., Wyder, S., Huerta-Cepas, J., . . Mering, C. V. (2019). STRING v11: protein-protein association networks with increased coverage, supporting functional discovery in genome-wide experimental datasets. Nucleic Acids Res, 47(D1), D607-d613. doi: 10.1093/nar/gky1131

Tabe, Y., Jin, L., Tsutsumi-Ishii, Y., Xu, Y., McQueen, T., Priebe, W., . . Konopleva, M. (2007). Activation of integrin-linked kinase is a critical prosurvival pathway induced in leukemic cells by bone marrow-derived stromal cells. Cancer Res, 67(2), 684-694. doi: 10.1158/0008-5472.Can-06-3166

Teague, R. M., \& Kline, J. (2013). Immune evasion in acute myeloid leukemia: current concepts and future directions. J Immunother Cancer, 1(13), 1. doi: 10.1186/2051-1426-1-13

Thorsson, V., Gibbs, D. L., Brown, S. D., Wolf, D., Bortone, D. S., Ou Yang, T. H., . . Shmulevich, I. (2018). The Immune Landscape of Cancer. Immunity, 48(4), 812-830 e814. doi: 10.1016/j.immuni.2018.03.023

Trott, O., \& Olson, A. J. (2010 Jan 30). AutoDock Vina: improving the speed and accuracy of docking with a new scoring function, efficient optimization, and multithreading. J Comput Chem, 31(2)(1096-987X (Electronic)), 455-461. doi: 10.1002/jcc.21334

Vadakekolathu, J., Minden, M. D., Hood, T., Church, S. E., Reeder, S., Altmann, H., . . Rutella, S. (2020). Immune landscapes predict chemotherapy resistance and immunotherapy response in acute myeloid leukemia. Sci Transl Med, 12(546). doi: 10.1126/scitranslmed.aaz0463

Walter, W., Sánchez-Cabo, F., \& Ricote, M. (2015). GOplot: an R package for visually combining expression data with functional analysis. Bioinformatics, 31(17), 2912-2914. doi: 


\subsection{3/bioinformatics/btv300}

Wegiel, B., Ekberg, J., Talasila, K. M., Jalili, S., \& Persson, J. L. (2009). The role of VEGF and a functional link between VEGF and p27Kip1 in acute myeloid leukemia. Leukemia, 23(2), 251-261. doi: 10.1038/leu.2008.300

Wu, Q., Liu, P., Lao, G., Liu, Y., Zhang, W., \& Ma, C. (2020). Comprehensive Analysis of circRNA-miRNA-mRNA Network in Cervical Squamous Cell Carcinoma by Integrated Analysis. Onco Targets Ther, 13, 8641-8650. doi: 10.2147/ott.S254323

Xiao, X., Mruk, D. D., \& Cheng, C. Y. (2013). Intercellular adhesion molecules (ICAMs) and spermatogenesis. Hum Reprod Update, 19(2), 167-186. doi: 10.1093/humupd/dms049

Xue, Y., Ge, Y., Kang, M., Wu, C., Wang, Y., Rong, L., \& Fang, Y. (2019). Selection of three miRNA signatures with prognostic value in non-M3 acute myeloid leukemia. BMC Cancer, 19(1), 109-109. doi: 10.1186/s12885-019-5315-Z

Yan, H., Qu, J., Cao, W., Liu, Y., Zheng, G., Zhang, E., \& Cai, Z. (2019). Identification of prognostic genes in the acute myeloid leukemia immune microenvironment based on TCGA data analysis. Cancer Immunol Immunother, 68(12), 1971-1978. doi: 10.1007/s00262-019-02408-7

Ye, H., Adane, B., Khan, N., Sullivan, T., Minhajuddin, M., Gasparetto, M., . . Jordan, C. T. (2016). Leukemic Stem Cells Evade Chemotherapy by Metabolic Adaptation to an Adipose Tissue Niche. Cell Stem Cell, 19(1), 23-37. doi: 10.1016/j.stem.2016.06.001

Yoshihara, K., Shahmoradgoli, M., Martínez, E., Vegesna, R., Kim, H., Torres-Garcia, W., . . Verhaak, R. G. (2013). Inferring tumour purity and stromal and immune cell admixture from expression data. Nat Commun, 4, 2612. doi: 10.1038/ncomms3612

Zhang, H., Pang, Y., Ma, C., Li, J., Wang, H., \& Shao, Z. (2018). ClC5 Decreases the Sensitivity of Multiple Myeloma Cells to Bortezomib via Promoting Prosurvival Autophagy. Oncol Res, 26(3), 421-429. doi: 10.3727/096504017X15049221237147 
bioRxiv preprint doi: https://doi.org/10.1101/2021.09.15.460430; this version posted September 16, 2021. The copyright holder has placed

this preprint (which was not certified by peer review) in the Public Domain. It is no longer restricted by copyright. Anyone can legally share, reuse, remix, or adapt this material for any purpose without crediting the original authors.

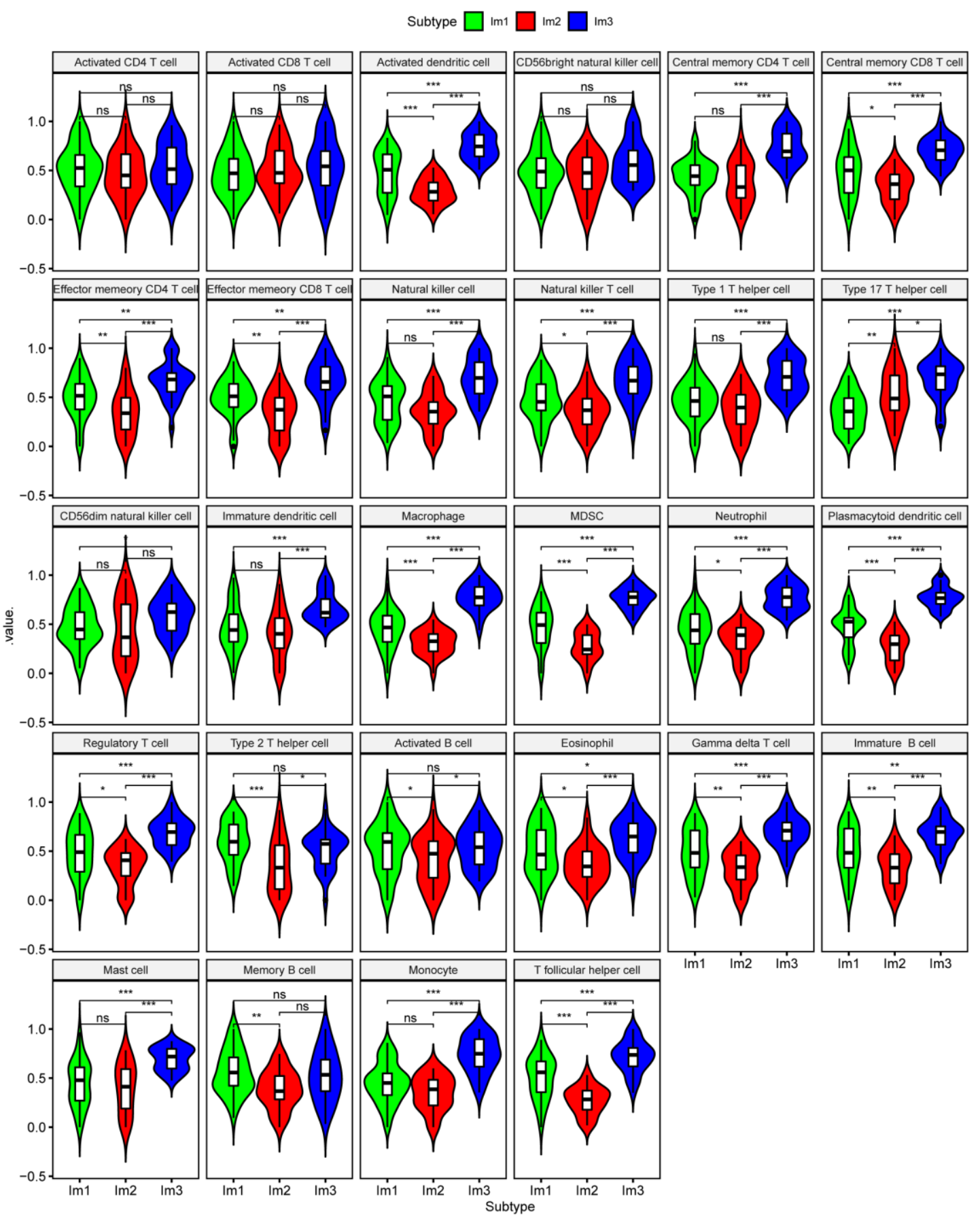

661 Figure S1. Comparison of the infiltration of immune cells in $\operatorname{Im} 1, \operatorname{Im} 2$ and $\operatorname{Im} 3$ clusters of

AML patients.

663 


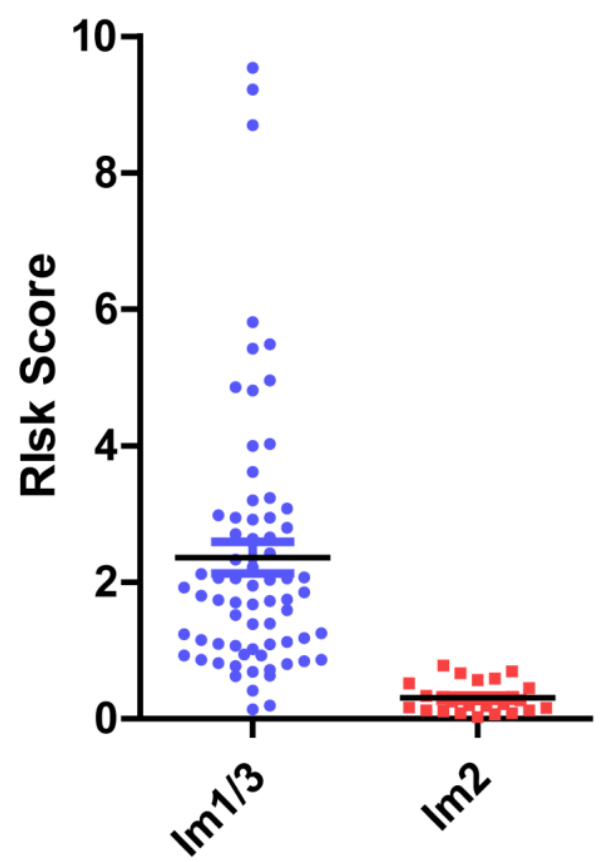

\section{Cluster}

665 Figure S2. Comparison of the risk score between Im2 (immune infiltration-lacking subtype,

IL type) and Im1/3(immune infiltration-rich subtype, IR type) clusters. 
A
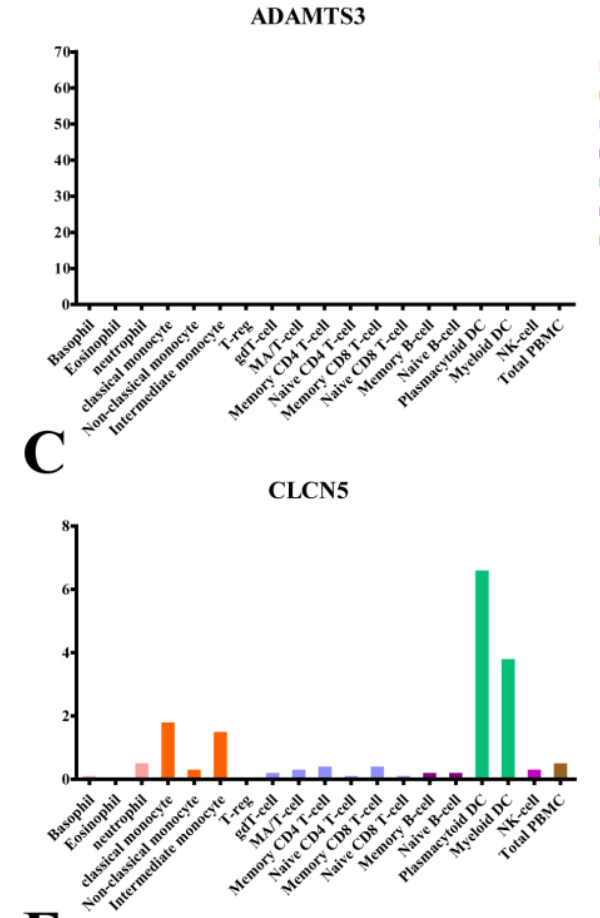

E

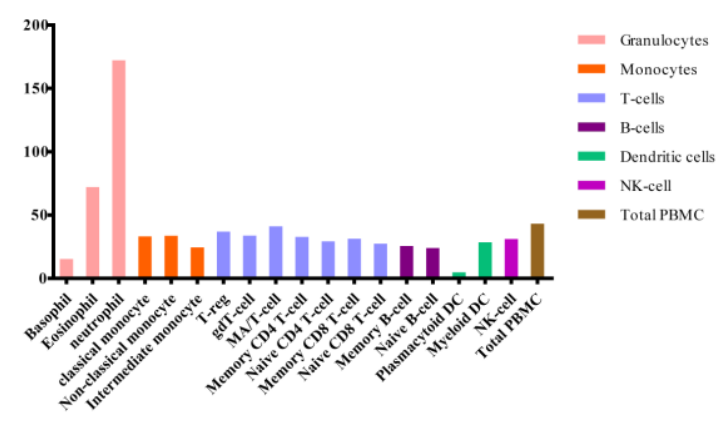

B
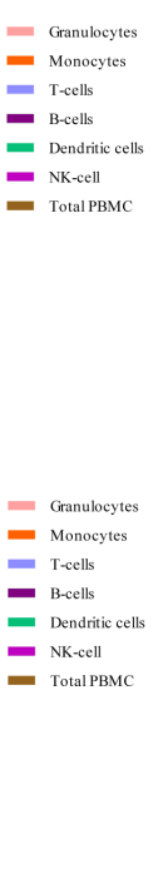

D

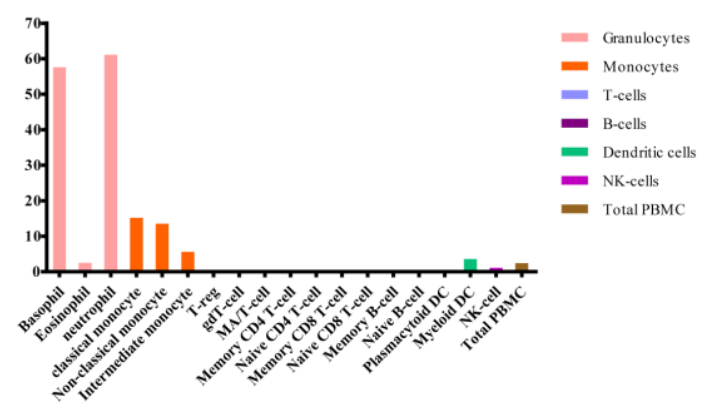

Figure S3. Histogram of hub gene expression in immune cells based on the Atlas database. 
672 Table S1. Clinical characters of TCGA-AML patients.

\begin{tabular}{|c|c|c|c|c|}
\hline ID & Gender & Age & OS & Status \\
\hline TCGA-AB-2808 & male & 23 & 95.4 & alive \\
\hline TCGA-AB-2819 & female & 52 & 83.2 & alive \\
\hline TCGA-AB-2821 & male & 64 & 27.4 & dead \\
\hline TCGA-AB-2828 & male & 55 & 76.1 & alive \\
\hline TCGA-AB-2835 & male & 48 & 55.8 & alive \\
\hline TCGA-AB-2846 & female & 57 & 46.7 & dead \\
\hline TCGA-AB-2847 & male & 53 & 20.3 & dead \\
\hline TCGA-AB-2849 & male & 39 & 74.0 & alive \\
\hline TCGA-AB-2862 & female & 33 & 47.7 & alive \\
\hline TCGA-AB-2863 & male & 63 & 1.0 & dead \\
\hline TCGA-AB-2869 & female & 64 & 8.1 & alive \\
\hline TCGA-AB-2870 & male & 76 & 5.1 & dead \\
\hline TCGA-AB-2871 & male & 51 & 5.1 & alive \\
\hline TCGA-AB-2872 & male & 42 & 21.3 & alive \\
\hline TCGA-AB-2873 & female & 51 & 9.1 & alive \\
\hline TCGA-AB-2874 & male & 59 & 13.2 & alive \\
\hline TCGA-AB-2875 & male & 43 & 7.1 & alive \\
\hline TCGA-AB-2876 & female & 45 & 40.6 & alive \\
\hline TCGA-AB-2877 & female & 60 & 21.3 & alive \\
\hline TCGA-AB-2878 & female & 47 & 12.2 & dead \\
\hline TCGA-AB-2880 & male & 24 & 14.1 & dead \\
\hline TCGA-AB-2881 & female & 48 & 13.1 & alive \\
\hline TCGA-AB-2882 & female & 73 & 12.2 & dead \\
\hline TCGA-AB-2883 & male & 60 & 24.3 & alive \\
\hline TCGA-AB-2884 & female & 44 & 24.4 & dead \\
\hline TCGA-AB-2885 & male & 71 & 7.1 & dead \\
\hline TCGA-AB-2886 & male & 26 & 6.0 & alive \\
\hline TCGA-AB-2888 & male & 57 & 11.1 & alive \\
\hline TCGA-AB-2889 & male & 55 & 10.1 & alive \\
\hline TCGA-AB-2892 & female & 42 & 31.4 & alive \\
\hline TCGA-AB-2894 & female & 50 & 6.0 & dead \\
\hline TCGA-AB-2895 & female & 41 & 5.1 & dead \\
\hline TCGA-AB-2896 & female & 21 & 7.1 & dead \\
\hline TCGA-AB-2897 & female & 50 & 8.1 & alive \\
\hline TCGA-AB-2898 & female & 69 & 13.1 & alive \\
\hline TCGA-AB-2899 & female & 76 & 22.4 & dead \\
\hline TCGA-AB-2900 & male & 70 & 6.1 & dead \\
\hline TCGA-AB-2901 & male & 27 & 2.0 & alive \\
\hline TCGA-AB-2908 & male & 81 & 1.0 & dead \\
\hline
\end{tabular}




\begin{tabular}{|c|c|c|c|c|}
\hline TCGA-AB-2911 & female & 51 & 39.5 & alive \\
\hline TCGA-AB-2912 & male & 63 & 9.1 & dead \\
\hline TCGA-AB-2913 & male & 61 & 40.5 & alive \\
\hline TCGA-AB-2914 & female & 22 & 26.4 & conse \\
\hline TCGA-AB-2916 & female & 48 & 29.4 & alive \\
\hline TCGA-AB-2917 & female & 41 & 40.5 & alive \\
\hline TCGA-AB-2919 & female & 54 & 2.0 & alive \\
\hline TCGA-AB-2920 & male & 44 & 12.2 & dead \\
\hline TCGA-AB-2924 & male & 59 & 3.0 & alive \\
\hline TCGA-AB-2925 & male & 57 & 8.1 & dead \\
\hline TCGA-AB-2927 & female & 88 & 3.0 & dead \\
\hline TCGA-AB-2928 & female & 43 & 2.0 & dead \\
\hline TCGA-AB-2929 & female & 71 & 4.1 & dead \\
\hline TCGA-AB-2933 & male & 58 & 4.1 & dead \\
\hline TCGA-AB-2934 & male & 65 & 0.9 & alive \\
\hline TCGA-AB-2935 & male & 66 & 2.0 & dead \\
\hline TCGA-AB-2936 & female & 61 & 2.0 & alive \\
\hline TCGA-AB-2937 & female & 36 & 7.2 & dead \\
\hline TCGA-AB-2939 & male & 72 & 15.2 & ve \\
\hline TCGA-AB-2942 & female & 67 & 21.4 & alive \\
\hline TCGA-AB-2948 & male & 67 & 19.3 & dead \\
\hline TCGA-AB-2949 & male & 58 & 23.3 & alive \\
\hline TCGA-AB-2950 & female & 34 & 10.2 & alive \\
\hline TCGA-AB-2952 & female & 60 & 1.0 & dead \\
\hline TCGA-AB-2955 & female & 56 & 16.3 & dead \\
\hline TCGA-AB-2956 & male & 61 & 6.1 & dead \\
\hline TCGA-AB-2959 & male & 71 & 16.3 & dead \\
\hline TCGA-AB-2963 & male & 56 & 54.7 & dead \\
\hline TCGA-AB-2965 & male & 60 & 11.2 & ad \\
\hline TCGA-AB-2966 & female & 57 & 28.5 & dead \\
\hline TCGA-AB-2970 & female & 34 & 10.2 & dead \\
\hline TCGA-AB-2971 & female & 76 & 26.4 & ad \\
\hline TCGA-AB-2973 & female & 68 & 20.3 & dead \\
\hline TCGA-AB-2976 & male & 53 & 30.5 & dead \\
\hline TCGA-AB-2977 & female & 71 & 1.0 & dead \\
\hline TCGA-AB-2979 & female & 30 & 22.4 & alive \\
\hline TCGA-AB-2980 & male & 50 & 23.3 & alive \\
\hline TCGA-AB-2981 & female & 35 & 16.2 & \\
\hline TCGA-AB-2982 & female & 29 & 5.0 & alive \\
\hline TCGA-AB-2983 & male & 45 & 11.2 & dead \\
\hline TCGA-AB-2984 & male & 38 & 38.6 & \\
\hline TCGA-AB-2986 & female & 31 & 7.1 & \\
\hline TCGA-AB-2987 & female & 75 & 6.1 & \\
\hline
\end{tabular}



reuse, remix, or adapt this material for any purpose without crediting the original authors.

$\begin{array}{lcccl}\text { TCGA-AB-2988 } & \text { female } & 67 & 1.0 & \text { dead } \\ \text { TCGA-AB-2990 } & \text { male } & 51 & 15.2 & \text { alive } \\ \text { TCGA-AB-2991 } & \text { female } & 40 & 60.9 & \text { alive } \\ \text { TCGA-AB-2992 } & \text { female } & 32 & 56.9 & \text { dead } \\ \text { TCGA-AB-2995 } & \text { male } & 63 & 51.7 & \text { alive } \\ \text { TCGA-AB-2996 } & \text { male } & 74 & 52.7 & \text { alive } \\ \text { TCGA-AB-2998 } & \text { female } & 68 & 1.0 & \text { dead } \\ \text { TCGA-AB-2999 } & \text { male } & 62 & 57.8 & \text { alive } \\ \text { TCGA-AB-3001 } & \text { female } & 31 & 52.7 & \text { alive } \\ \text { TCGA-AB-3002 } & \text { male } & 68 & 47.7 & \text { dead } \\ \text { TCGA-AB-3007 } & \text { male } & 35 & 52.7 & \text { alive } \\ \text { TCGA-AB-3008 } & \text { male } & 22 & 27.4 & \text { dead } \\ \text { TCGA-AB-3009 } & \text { male } & 23 & 19.2 & \text { dead } \\ \text { TCGA-AB-3011 } & \text { female } & 21 & 62.8 & \text { alive } \\ \text { TCGA-AB-3012 } & \text { male } & 53 & 62.9 & \text { alive }\end{array}$

673 
bioRxiv preprint doi: https://doi.org/10.1101/2021.09.15.460430; this version posted September 16, 2021. The copyright holder has placed this preprint (which was not certified by peer review) in the Public Domain. It is no longer restricted by copyright. Anyone can legally share, reuse, remix, or adapt this material for any purpose without crediting the original authors.

675 Table S2. 98 survival-related immune genes (SIGs).

\begin{tabular}{|c|c|}
\hline SIGs & p-value \\
\hline ADAM12 & 0.009055077 \\
\hline ADAMTS3 & 0.019672714 \\
\hline ADGRE5 & 0.030478533 \\
\hline AHNAK & 0.018578223 \\
\hline AIF1 & 0.01115535 \\
\hline AKT3 & 0.029314184 \\
\hline ANXA5 & 0.041670163 \\
\hline APOL3 & 0.012945343 \\
\hline ARHGAP10 & 0.003128417 \\
\hline ARL1 & 0.019752628 \\
\hline ASB2 & 0.005555865 \\
\hline ATP6V1A & 0.040023492 \\
\hline BST2 & 0.017630636 \\
\hline C1orf162 & 0.024263467 \\
\hline C1 orf54 & 0.014255787 \\
\hline CCR5 & 0.013876728 \\
\hline CD109 & 0.012251905 \\
\hline CD37 & 0.000151561 \\
\hline $\mathrm{CD} 4$ & 0.013047345 \\
\hline CD52 & 0.000148262 \\
\hline CD79B & 0.047103955 \\
\hline CFL1 & 0.028244461 \\
\hline CLCN5 & 0.0000025 \\
\hline CLIC2 & 0.012485077 \\
\hline CLIC3 & 0.025449448 \\
\hline CREB5 & 0.024746533 \\
\hline CRYBB1 & 0.031592817 \\
\hline CSF2RA & 0.040604671 \\
\hline CTPS1 & 0.006880889 \\
\hline CTSD & 0.016154468 \\
\hline CXCR2 & 0.039440985 \\
\hline CYP27A1 & 0.032368574 \\
\hline CYTH1 & 0.010866987 \\
\hline DAB2 & 0.030817755 \\
\hline DAPK1 & 0.046970159 \\
\hline DARS & 0.009274375 \\
\hline DAXX & 0.000084 \\
\hline DPYD & 0.013317327 \\
\hline EMP3 & 0.007094884 \\
\hline
\end{tabular}


F12

FABP5

FAM49A

FERMT3

GAB3

HAL

HCP5

HELLS

HNMT

HRH1

HSPA6

ICAM3

IDH3A

IGFBP5

IKZF1

IL3RA

INPP5F

ITGAL

ITGAM

ITGB2

ITGB4

KIR2DL1

KIR3DL2

KLRG1

LAMP2

LGALS1

LRRC42

LSP1

LST1

MAK

MBP

MMD

MOCOS

NCAM1

NUCB2

OFD1

PDCD1

PIK3IP1

PLA2G4A

PNPLA6

PTGES2

PTGS1

RPS9
0.03259722

0.004115137

0.021102459

0.000482707

0.012790197

0.030493772

0.005447746

0.030883597

0.016554784

0.015225832

0.030522513

0.048885585

0.034302608

0.031599509

0.03136533

0.030153684

0.003711848

0.002788804

0.036646269

0.030734879

0.020982102

0.011560605

0.048387167

0.015460397

0.013655645

0.000181036

0.020273562

0.000744407

0.015009468

0.030356179

0.038905732

0.017479911

0.004815371

0.009065868

0.00788809

0.022159756

0.021997507

0.006985001

0.033592713

0.007064373

0.035224203

0.002302456

0.026383806 
bioRxiv preprint doi: https://doi.org/10.1101/2021.09.15.460430; this version posted September 16, 2021. The copyright holder has placed this preprint (which was not certified by peer review) in the Public Domain. It is no longer restricted by copyright. Anyone can legally share, reuse, remix, or adapt this material for any purpose without crediting the original authors.

$\begin{array}{cc}\text { RUBCN } & 0.001673805 \\ \text { S100A4 } & 0.000139415 \\ \text { SELE } & 0.004005489 \\ \text { SFXN3 } & 0.001168979 \\ \text { SIGLEC10 } & 0.036557072 \\ \text { SLA } & 0.027371484 \\ \text { SPCS3 } & 0.033123296 \\ \text { ST3GAL6 } & 0.022176552 \\ \text { ST8SIA4 } & 0.045818496 \\ \text { TACSTD2 } & 0.025528048 \\ \text { TLR9 } & 0.03758144 \\ \text { TNFAIP2 } & 0.001039854 \\ \text { TOGARAM2 } & 0.030739985 \\ \text { TOX4 } & 0.034453285 \\ \text { UPP1 } & 0.046621377 \\ \text { VCAM1 } & 0.030889137\end{array}$

